\title{
Low scale left-right symmetry and naturally small neutrino mass
}

\author{
Vedran Brdar and Alexei Yu. Smirnov \\ Max-Planck-Institut für Kernphysik, \\ 69117 Heidelberg, Germany \\ E-mail: vbrdar@mpi-hd.mpg.de, smirnov@mpi-hd.mpg.de
}

ABSTRACT: We consider the low scale (10-100 TeV) left-right symmetric model with "naturally" small neutrino masses generated through the inverse seesaw mechanism. The Dirac neutrino mass terms are taken to be similar to the masses of charged leptons and quarks in order to satisfy the quark-lepton similarity condition. The inverse seesaw implies the existence of fermion singlets $S$ with Majorana mass terms as well as the "left" and "right" Higgs doublets. These doublets provide the portal for $S$ and break the left-right symmetry. The inverse seesaw allows to realize a scenario in which the large lepton mixing originates from the Majorana mass matrix of $S$ fields which has certain symmetry. The model contains heavy pseudo-Dirac fermions, formed by $S$ and the right-handed neutrinos, which have masses in the $1 \mathrm{GeV}-100 \mathrm{TeV}$ range and can be searched for at current and various future colliders such as LHC, FCC-ee and FCC-hh as well as in SHiP and DUNE experiments. Their contribution to neutrinoless double beta decay is unobservable. The radiative corrections to the mass of the Higgs boson and the possibility for generating the baryon asymmetry of the Universe are discussed. Modification of the model with two singlets $\left(S_{L}\right.$ and $S_{R}$ ) per generation can provide a viable keV-scale dark matter candidate.

Keywords: Beyond Standard Model, Neutrino Physics

ArXiv EPrint: 1809.09115 


\section{Contents}

1 Introduction 1

2 The model and neutrino masses 3

2.1 The model, linear and inverse seesaw 3

$\begin{array}{lll}2.2 & \text { Screening and } q-l \text { similarity } & 7\end{array}$

2.3 Flavor symmetries 8

3 Phenomenology and naturalness $\quad 9$

3.1 Heavy neutral lepton searches $\quad 9$

$3.20 \nu 2 \beta$ decay 13

$\begin{array}{lll}3.3 & \text { Leptogenesis } & 14\end{array}$

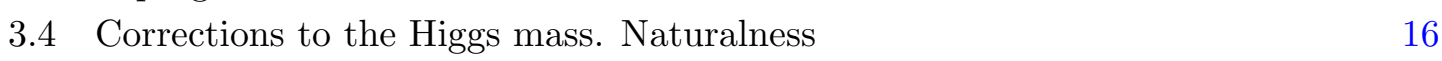

4 Variation on the theme $\quad 17$

$\begin{array}{ll}\text { 4.1 Altering heavy fermion portal couplings } & 17\end{array}$

$\begin{array}{lll}4.2 & \text { Left and right fermion singlets } & 19\end{array}$

$\begin{array}{llr}5 & \text { Summary } & 22\end{array}$

\section{Introduction}

The left-right symmetric models [1-5] based on the gauge symmetry group

$$
\mathrm{SU}(2)_{L} \times \mathrm{SU}(2)_{R} \times \mathrm{U}(1)_{B-L},
$$

and parity $P$ which ensures the equality between couplings in the left and right sectors, are still one of the most appealing and well-motivated extensions of the Standard Model $(\mathrm{SM})$ [6-15]. Spontaneous breaking of $\mathrm{SU}(2)_{R} \times \mathrm{U}(1)_{B-L} \times P$ down to the SM symmetry group explains the observed low-energy asymmetry between the left and right as well as provides a natural framework for the generation of small neutrino masses via the seesaw mechanism [16-19]. The scale of left-right (L-R) symmetry breaking and the seesaw scale coincide. The key question is whether this scale can be at $\mathcal{O}(10-100) \mathrm{TeV}$ energies that are accessible to LHC and the next generation of colliders.

The majority of the low scale L-R symmetric models constructed so far is at odds with the generation of "naturally" small neutrino masses. In what follows we will call the neutrino mass to be naturally small if mechanism of its generation employs the Dirac neutrino masses, $m_{\nu}^{D}$, similar in size to the Dirac masses of charged leptons, $m_{l}$, and quarks, $m_{q}$, i.e.

$$
m_{\nu}^{D} \approx m_{q}, m_{l} .
$$


This relation facilitates the grand unification and we will refer to it as to the quark-lepton $(q-l)$ similarity condition. The usual type-I seesaw mechanism realizes such a possibility provided that the scale of right-handed ( $\mathrm{RH})$ neutrino masses is about $10^{14} \mathrm{GeV}$. Lowering the scale of RH neutrinos down to e.g. $10^{4} \mathrm{GeV}$ requires $m_{\nu}^{D} \sim 1 \mathrm{MeV}$, which is 5 orders of magnitude smaller than the top quark mass and thus is not in accord with eq. (1.2) for the third generation of neutrinos. If neutrinos acquire their masses dominantly via the type-II seesaw mechanism, the Dirac mass terms should be even more strongly suppressed.

In this paper, in order to reconcile the low scale L-R symmetry and the naturally small neutrino masses we assume that the latter are generated via the inverse seesaw mechanism $[20,21]$. In such a framework, the small neutrino masses can be obtained for values of the Dirac mass terms that are in accord with eq. (1.2). The inverse seesaw mechanism requires the introduction of new fermionic singlets, $S$, which couple to the $\mathrm{RH}$ neutrinos and thus form the Dirac mass terms. We introduce three such singlets (one per generation), whose Majorana masses are much smaller than the electroweak scale. The generation of light neutrino masses via the inverse seesaw requires the right-handed $\mathrm{SU}(2)_{R}$ Higgs doublet and therefore, due to L-R symmetry, the left-handed $\mathrm{SU}(2)_{L}$ doublet. These doublets break the L-R symmetry, so that the Higgs triplets are not needed [22-24]. Hence, the problem of the absence of low-dimensional representations ${ }^{1}$ in the model does not appear.

We do not assume any special smallness of the Yukawa couplings of the scalar doublets. These couplings are similar or even equal to the couplings of the bi-doublet. If equal, the so called screening of the Dirac structures is realized [25], and the large lepton mixing originates from a certain structure of the Majorana mass matrix of singlets $S$ (the screening was previously studied for the double seesaw mechanism [26]). The mass matrix of $S$ may have certain symmetry which leads, e.g., to the tribimaximal mixing.

In this paper we elaborate on such a scenario. We focus on generation of neutrino mass and mixing in the L-R model with Higgs doublets instead of triplets. Such models have been extensively explored before $[4,27]$ and the only new element here are singlet fermions $S$ which allow to realize the inverse seesaw mechanism (as a dominant mechanism for the generation of neutrino mass) and certain selection of the Yukawa couplings. We explore here new features related to introduction of the fermion singlets, while the gauge and scalar boson sectors are the same as in several earlier publications. We confront the model with the existing experimental data from the beam-dump experiments, LHC, experiments searching for neutrinoless double beta decay, etc., and also estimate the discovery potential of future colliders and neutrino oscillation facilities. We obtain bounds on relevant parameters and constrain the L-R symmetry breaking scale. We examine the possibility for the generation of the observed baryon asymmetry of the Universe and address the issue of the Higgs naturalness. We also consider the extensions of the aforementioned scenario, in particular the scenario with two $S$ fields (left and right) per generation which contains a viable dark matter candidate.

\footnotetext{
${ }^{1}$ In the vast majority of studies, L-R symmetry is broken by introducing the Higgs triplets, while the Higgs doublets are absent. The existence of the higher representations (triplets) and the absence of lowdimensional representations (doublets here) should have a certain reason and a proper physical explanation.
} 
The paper is organized as follows. In section 2, we describe the model and generation of the neutrino masses, and discuss the possibility to introduce flavor symmetries. The phenomenology of the model is presented in section 3. We elaborate on the various extensions of this scenario in section 4 . Finally, in section 5 we conclude.

\section{The model and neutrino masses}

\subsection{The model, linear and inverse seesaw}

Leptons are organized in the following representations of the symmetry group (1.1)

$$
L_{L}=\left(\begin{array}{c}
\nu_{l} \\
l
\end{array}\right)_{L} \sim(2,1,-1), \quad L_{R}=\left(\begin{array}{c}
\nu_{l} \\
l
\end{array}\right)_{R} \sim(1,2,-1), \quad S \sim(1,1,0),
$$

where $l=\{e, \mu, \tau\}$ and in brackets we indicate the corresponding quantum numbers. The Majorana leptons $S$ are complete gauge singlets. We assume the existence of three such leptons - one per generation.

The scalar sector consists of the usual bi-doublet

$$
\Phi=\left(\begin{array}{cc}
\phi_{1}^{0} & \phi_{2}^{+} \\
\phi_{1}^{-} & \phi_{2}^{0}
\end{array}\right) \sim(2,2,0)
$$

and two doublets

$$
\chi_{L}=\left(\begin{array}{c}
\chi_{L}^{+} \\
\chi_{L}^{0}
\end{array}\right) \sim(2,1,1), \quad \chi_{R}=\left(\begin{array}{c}
\chi_{R}^{+} \\
\chi_{R}^{0}
\end{array}\right) \sim(1,2,1) .
$$

The latter are required for the realization of the inverse seesaw mechanism as well as for the L-R symmetry breaking. In the first step, the neutral component of the right-handed scalar doublet $\chi_{R}$ obtains a non-vanishing vacuum expectation value (VEV), which breaks the $\mathrm{SU}(2)_{R} \times \mathrm{U}(1)_{B-L}$ down to $\mathrm{U}(1)_{Y}$, where $Y$ is the hypercharge. In the second step, the neutral scalar fields from left-handed scalar doublet $\chi_{L}\left(\chi_{L}^{0}\right)$ and bi-doublet $\Phi\left(\phi_{1}^{0}, \phi_{2}^{0}\right)$ break the $\mathrm{SU}(2)_{L} \times \mathrm{U}(1)_{Y}$ symmetry down to $\mathrm{U}(1)_{\mathrm{EM}}$. Their VEVs should satisfy the relation

$$
\sqrt{\left\langle\chi_{L}^{0}\right\rangle^{2}+\left\langle\phi_{1}^{0}\right\rangle^{2}+\left\langle\phi_{2}^{0}\right\rangle^{2}} \approx 246 \mathrm{GeV}
$$

in order to reproduce the electroweak scale. Notice that for the electroweak symmetry breaking only one non-zero VEV among these three neutral fields is enough. The L-R symmetry breaking requires

$$
\left\langle\chi_{R}^{0}\right\rangle \gg\left\langle\chi_{L}^{0}\right\rangle,\left\langle\phi_{1,2}^{0}\right\rangle \text {. }
$$

The Higgs sector in eqs. (2.2) and (2.3) is identical to the one in earlier publications $[4,27]$. Minimization of the potential had been done under certain simplifications. In particular, it was assumed a priori that the electic charge is conserved in minimum. The tri-linear terms in potential are absent due to additional symmetry. With such conditions, it was shown $[4,27]$ that the global minimum exists for a certain range of parameters of the potential, in which the inequality (2.5) is satisfied, i.e. the parity is spontaneosly 
broken. The requirements for such a scenario are inequalities of certain quartic couplings and positivity or small values of other couplings [4, 27]. The values of VEVs are controlled (at least in the case of mild hierarchy) by the mass parameters for the doublets and bi-doublet. For the most general form of the potential (and without above discussed assumptions) the minimization has not been done for the bi-doublet-doublet case. Such a study has been done recently [28] for the bi-doublet-triplet scenario and some information can be inferred from the obtained results. It has been found in [28] that there are significant regions in the parameter space where required minimum can be obtained. Note that bi-doublet-doublet and bi-doublet-triplet models have the same number of neutral bosons, and structure of the terms in the potential is similar. Interestingly, the $\beta$ - terms in the bi-doublet-triplet potential should be small in the minimum and the terms of similar type in the bidoublet-doublet potential are absent.

For the rest of our paper it is enough that hierarchy (2.5) is achieved at least for some choice of parameters. Since we are not discussing phenomenology of the Higgs sector (masses of scalars, decay rates, etc.) specific values of the parameters of the higgs potential are not important.

The lepton masses are generated by the following Lagrangian [22]

$$
\mathcal{L} \supset-\bar{L}_{R} Y \Phi^{\dagger} L_{L}-\bar{L}_{R} \tilde{Y} \tilde{\Phi}^{\dagger} L_{L}-\bar{S} Y_{L} \tilde{\chi}_{L}^{\dagger} L_{L}-\bar{S}^{c} Y_{R} \tilde{\chi}_{R}^{\dagger} L_{R}-\frac{1}{2} \bar{S}^{c} \mu S+\text { h.c. },
$$

where $Y, \tilde{Y}, Y_{L}, Y_{R}$ are $3 \times 3$ matrices of the Yukawa couplings and $\mu$ is the $3 \times 3$ Majorana mass matrix of $S$ leptons. $\tilde{X} \equiv i \sigma_{2} X^{*}\left(X=\left\{\chi_{L}, \chi_{R}\right\}\right), S^{c} \equiv C \bar{S}^{T}$ and $\tilde{\Phi} \equiv \sigma_{2} \Phi^{*} \sigma_{2}$ denote charge conjugated fields of scalars and fermions. The field transformations under parity

$$
L_{L} \Longleftrightarrow L_{R}, \quad \chi_{L} \Longleftrightarrow \chi_{R}, \Phi \Longleftrightarrow \Phi^{\dagger}, \quad S \Longleftrightarrow S^{c},
$$

impose the following relations

$$
Y_{L}=Y_{R}, \quad Y=Y^{\dagger}, \quad \tilde{Y}=\tilde{Y}^{\dagger}, \quad \mu=\mu^{\dagger},
$$

above the L-R symmetry breaking scale.

When the scalar fields acquire VEVs, the interactions (2.6) generate the mass matrix of neutral leptons

$$
\mathcal{M}=\left(\begin{array}{ccc}
0 & m_{D}^{T} & m_{D}^{\prime T} \\
m_{D} & 0 & M_{D}^{T} \\
m_{D}^{\prime} & M_{D} & \mu
\end{array}\right),
$$

given in the $\left(\nu_{L}, N_{L}, S^{c}\right)$ basis $\left(N_{L} \equiv \nu_{R}^{c}\right)$. Here,

$$
m_{D}=\frac{1}{\sqrt{2}}\left(Y\left\langle\phi_{1}^{0}\right\rangle+\tilde{Y}\left\langle\phi_{2}^{0}\right\rangle\right), \quad m_{D}^{\prime}=\frac{1}{\sqrt{2}} Y_{L}\left\langle\chi_{L}^{0}\right\rangle, \quad M_{D}=\frac{1}{\sqrt{2}} Y_{R}\left\langle\chi_{R}^{0}\right\rangle .
$$

For simplicity we assume $\left\langle\phi_{1}^{0}\right\rangle \gg\left\langle\phi_{2}^{0}\right\rangle$ such that only the first term in $m_{D}$ contributes.

The block diagonalization of $\mathcal{M}$ leads to the light neutrino mass matrix

$$
m_{\nu} \simeq \frac{\left\langle\chi_{L}^{0}\right\rangle}{\left\langle\chi_{R}^{0}\right\rangle}\left(m_{D}+m_{D}^{T}\right)-m_{D}^{T} M_{D}^{-1} \mu\left(M_{D}^{T}\right)^{-1} m_{D}
$$


Here, the first term is the linear seesaw contribution [20], whose existence is a generic consequence of the inverse seesaw realization in the L-R models. Notice that due to $Y_{L}=$ $Y_{R}$, the Yukawa matrices cancel and this term is given by the Dirac mass matrix multiplied by the ratio of VEVs of the two doublets. For $\left\langle\chi_{L}^{0}\right\rangle$ of the order of electroweak scale, and in the absence of unnaturally small elements of $m_{D}$, this term yields too large neutrino masses. Furthermore, if $m_{D} \propto m_{u}$ (the subscript $u$ denotes the up-type quarks), it has a wrong flavor structure with too strong mass hierarchy and small mixing. This is incompatible with the neutrino mass squared differences and large mixing angles observed in the oscillation experiments. Therefore, the linear seesaw contribution should be at most sub-dominant and the main contribution to the neutrino mass should arise from the inverse seesaw, given in the second term of eq. (2.11). Since $m_{D} \sim m_{u}$, this is achieved for

$$
\frac{\left\langle\chi_{L}^{0}\right\rangle}{\left\langle\chi_{R}^{0}\right\rangle}<\frac{0.05 \mathrm{eV}}{2 m_{D}^{\max }} \sim 10^{-12}
$$

where $m_{D}^{\max }$ denotes the largest entry of the Dirac neutrino mass matrix. It is worth noting that for $\left\langle\chi_{L}^{0}\right\rangle,\left\langle\phi_{2}^{0}\right\rangle \ll\left\langle\phi_{1}^{0}\right\rangle \approx 246 \mathrm{GeV}$, the SM Higgs boson is associated to the real part of $\phi_{1}^{0}$ field.

In order to estimate $\left\langle\chi_{L}^{0}\right\rangle$ we consider the following terms of the scalar potential

$$
V \supset h \chi_{L}^{\dagger} \tilde{\Phi} \chi_{R}-m_{\chi}^{2} \chi_{L}^{\dagger} \chi_{L}
$$

where $h$ is the dimensionful coupling and the term $\lambda\left(\chi_{L}^{\dagger} \chi_{L}\right)^{2}$ can be neglected for small values of $\left\langle\chi_{L}^{0}\right\rangle$. The minimization condition, $\partial V / \partial \chi_{L}^{\dagger}=0$, gives

$$
\left\langle\chi_{L}^{0}\right\rangle=h \frac{\left\langle\phi_{1}^{0}\right\rangle}{\left\langle\chi_{R}^{0}\right\rangle}
$$

where we have taken into account that due to the L-R symmetry $m_{\chi_{L}}^{2}=m_{\chi_{R}}^{2} \sim\left\langle\chi_{R}^{0}\right\rangle^{2}$. Using the condition (2.12) we obtain

$$
h \lesssim 40 \mathrm{keV}\left(\frac{\left\langle\chi_{R}^{0}\right\rangle}{10^{5} \mathrm{GeV}}\right)^{2},
$$

which needs to be satisfied in order to generate neutrino masses mainly via the inverse seesaw mechanism. Notice that eq. (2.14) is a realization of the VEV seesaw [29]. In contrast to the triplet case, the electroweak scale VEV (of the bi-doublet) enters this relation linearly and it contains dimensionful coupling $h$. According to eq. (2.14), the VEV of $\chi_{L}^{0}$ is controlled by free parameter $h$.

The coupling $h$ in the potential (15) can be forbidden by symmetry with respect to transformation $\Phi \rightarrow e^{i \pi / 2} \Phi[27]$. This symmetry is explicitly broken by the Yukawa interactions of $\Phi$, and therefore it does not prevent the appearance of the $h$ term in higher orders of perturbation theory. Even if the coupling $h$ vanishes at tree-level due to some symmetry, $\left\langle\chi_{L}^{0}\right\rangle \neq 0$ is generated radiatively via the one-loop diagram shown in figure 1 . It can be estimated as

$$
\left\langle\chi_{L}^{0}\right\rangle \simeq \frac{1}{16 \pi^{2}}\left\langle\chi_{R}^{0}\right\rangle \frac{\left\langle\phi_{1}^{0}\right\rangle}{\left\langle\chi_{R}^{0}\right\rangle} \frac{\mu}{\left\langle\chi_{R}^{0}\right\rangle}
$$




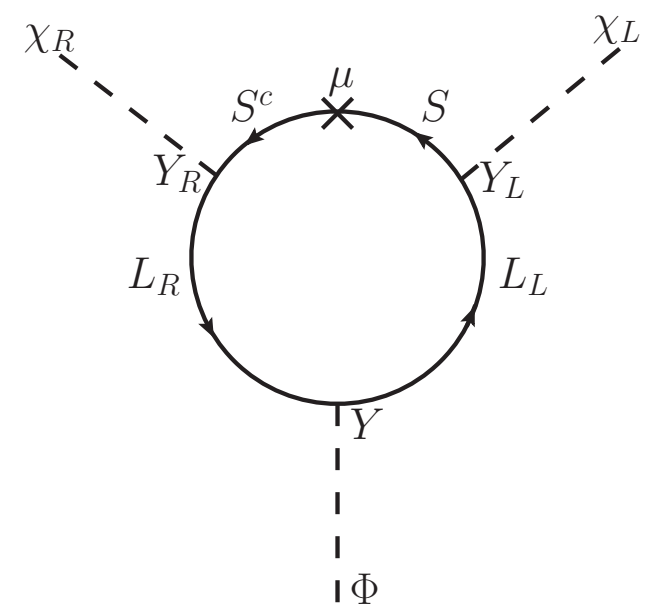

Figure 1. 1-loop diagram generating $\chi_{L}^{\dagger} \Phi \chi_{R}$ term in the potential.

For $\mu \simeq \mathcal{O}(10-100) \mathrm{keV}$ (see below) and $\left\langle\chi_{R}^{0}\right\rangle=10^{5} \mathrm{GeV}$ eq. (2.16) gives $\left\langle\chi_{L}^{0}\right\rangle \simeq 10^{-14}\left\langle\chi_{R}^{0}\right\rangle$ which satisfies eq. (2.12).

Notice that for $\left\langle\chi_{R}^{0}\right\rangle \sim 10^{5} \mathrm{GeV}$ (see section 3.1), $h$ should be at most $100 \mathrm{keV}$, which (as we will see) is of the order of $\mu$. This smallness can be associated to violation of the lepton number. In particular, one can reintroduce the global lepton number $L_{g}$ and assign the charges $(1,-1,1)$ to $\left(\nu_{L}, N_{L}, S\right)$. In the limit $m_{D}^{\prime}, \mu \rightarrow 0$, the conservation of $\tilde{L}$ is recovered and hence the small value of $\mu$ appears to be technically natural à la 't Hooft [30].

The inverse seesaw contribution in eq. (2.11) can be rewritten as

$$
m_{\nu} \approx \frac{\left\langle\phi_{1}^{0}\right\rangle^{2}}{\left\langle\chi_{R}^{0}\right\rangle^{2}} Y^{T} Y_{R}^{-1} \mu\left(Y_{R}^{T}\right)^{-1} Y
$$

For $Y_{3}, Y_{R 3}=1$ we obtain $m_{\nu} \approx\left(\left\langle\phi_{1}^{0}\right\rangle /\left\langle\chi_{R}^{0}\right\rangle\right)^{2} \mu$ (hereafter we denote Yukawa matrices and the corresponding Dirac mass terms with only one subscript since these matrices are taken to be diagonal (see section 2.3)) which allows us to estimate $\left\langle\chi_{R}^{0}\right\rangle$ as

$$
\left\langle\chi_{R}^{0}\right\rangle \simeq 3.5 \cdot 10^{5} \mathrm{GeV}\left(\frac{\mu}{100 \mathrm{keV}}\right)^{1 / 2}\left(\frac{0.05 \mathrm{eV}}{m_{\nu 3}}\right)^{1 / 2}
$$

where $\mu$ is the representative element of the corresponding matrix and $m_{\nu 3}$ is the largest active neutrino mass. Consequently,

$$
\mu_{i j} \ll m_{D i}, M_{D i} .
$$

The inverse seesaw not only explains the smallness of neutrino masses under the condition of $q-l$ similarity, but also provides a rather appealing framework for the lepton mixing generation where the large mixing angles originate from the $\mu$ matrix of singlets $S$, i.e. from the hidden sector.

The leptonic mixing matrix (PMNS) can be written as

$$
U_{\mathrm{PMNS}}=U_{l}^{\dagger} U_{\nu}
$$


where $U_{l}$ follows from the diagonalization of the charged lepton mass matrix, whereas $U_{\nu}$ diagonalizes the neutrino mass matrix (2.17) generated by the inverse seesaw mechanism. A good agreement with experimental data can be achieved if

$$
U_{l} \approx V_{\mathrm{CKM}}, \quad U_{\nu} \sim U_{\mathrm{TBM}} \text { or } U_{\mathrm{BM}},
$$

where $V_{\mathrm{CKM}}$ is the mixing matrix in the quark sector, and TBM and BM denote tribimaximal [31] and bimaximal [32, 33] mixing matrices. The first relation in eq. (2.21) could be a consequence of the grand unification or identical horizontal symmetry in the quark and lepton sectors. The second relation can follow from certain symmetry in the singlet sector.

\section{$2.2 \quad$ Screening and $q-l$ similarity}

Let us consider the generation of neutrino mixing in the basis where $Y$, and therefore the Dirac mass matrix $m_{D}$, are diagonal

$$
Y=Y^{\operatorname{diag}} \equiv \operatorname{diag}\left(Y_{1}, Y_{2}, Y_{3}\right) .
$$

First, we assume that

$$
Y_{R}=Y
$$

(and also $Y_{L}=Y$ ), so that $m_{D} \propto M_{D}$. This equality can be a consequence (a remnant) of further unification when $\nu_{L}, N_{L}$ and $S^{c}$ enter the same multiplet, e.g. 27-plet of the $E_{6}$ grand unification theory. This relation can also stem from certain horizontal symmetry [34]. The equality in eq. (2.23) leads to the screening of the Dirac structures: $Y_{R}$ and $Y$ cancel in the expression for the light neutrino mass matrix (see eq. (2.17)), so that

$$
m_{\nu} \approx \xi^{2} \mu,
$$

where

$$
\xi \equiv \frac{\left\langle\phi_{1}^{0}\right\rangle}{\left\langle\chi_{R}^{0}\right\rangle}=\frac{m_{D i}}{M_{D i}} .
$$

According to eq. (2.24), the structure of the light neutrino mass matrix is given by the structure of the Majorana matrix $\mu$. In particular, the neutrino contribution to the PMNS matrix is determined by $\mu$ :

$$
U_{\nu}^{T} m_{\nu} U_{\nu}=\xi^{2} U_{\nu}^{T} \mu U_{\nu}=\operatorname{diag}\left(m_{\nu 1}, m_{\nu 2}, m_{\nu 3}\right) .
$$

Previously, such a cancellation of the couplings has been considered for the double seesaw model in refs. [25, 26, 34, 35].

Second, we assume the $q-l$ similarity

$$
Y \approx Y_{u}
$$

where $Y_{u}$ is the up-type quark Yukawa coupling matrix. The screening and the $q-l$ similarity conditions determine the phenomenology of this scenario. 


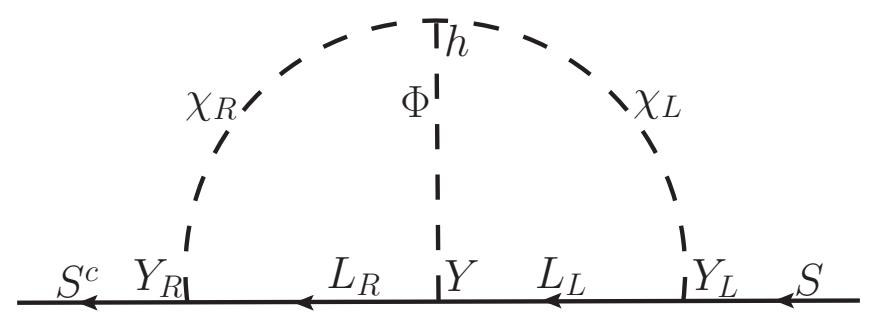

Figure 2. The leading radiative correction to the Majorana mass $\mu$.

\subsection{Flavor symmetries}

The matrices $Y$ and $Y_{R}$ can be diagonal simultaneously due to the $G_{\text {basis }}=Z_{2} \times Z_{2}$ symmetry with $(-,-),(+,-),(-,+)$ charges for the three generations of fermions and uncharged scalar sector. We will call $G_{\text {basis }}$ the basis fixing symmetry [34]. This symmetry is broken by the non-diagonal matrix $\mu$ and, in fact, the smallness of $\mu$ with respect to the other scales in the model can be related to this breaking. The $\mu$ term can arise from the interactions of $S$ with the new gauge singlet bosons which carry non-trivial $Z_{2} \times Z_{2}$ charge and develop non-zero VEVs. This Abelian symmetry, however, does not ensure the equality of the diagonal elements of $Y$ and $Y_{R}$. The equality can be achieved by introducing, for instance, a permutation symmetry or by further unification mentioned above.

In the case of the exact screening, the matrix $\mu$ should have nearly tribimaximal form in the basis fixed by $G_{\text {basis }}=Z_{2} \times Z_{2}$. This can be achieved by introducing the non-Abelian (e.g., discrete) symmetry $G_{f}$ which is broken down to $G_{\text {basis }}$ in the visible sector and to another residual symmetry $G_{\text {hidden }}=Z_{2} \times Z_{2}$ in the $S$-sector [34]. In the visible sector, $G_{f}$ can be broken explicitly. Similar construction has been realized for the double seesaw model [26] with masses of singlets at the Planck scale. In [26] the explicit symmetry breaking occurs at the lower (grand unification) scale and its impact on the neutrino mixing is suppressed by $\mathcal{O}\left(M_{\mathrm{GUT}} / M_{\mathrm{Pl}}\right)$ factor. In the case of inverse seesaw, the $\mu$ scale (with certain symmetry) is much lower than the explicit symmetry breaking scale $M_{D}$. So, a priori, the corrections to $\mu$ can be large, thus destroying the structure of $\mu$ imposed by symmetry. To check this, let us assume that the required structure of $\mu$, and consequently $m_{\nu}$, is achieved at the tree-level and estimate the corresponding radiative corrections. The lowest order correction to $\mu$ is given by the two loop diagram shown in figure 2. It can be estimated as

$$
\Delta \mu_{j j} \simeq \frac{1}{\left(16 \pi^{2}\right)^{2}} Y_{L j}^{*} Y_{R j} Y_{j} h
$$

The corrections are diagonal due to screening. The hierarchical values of Yukawa couplings $Y_{L j}, Y_{R j}, Y_{j}$ violate symmetry that sets the pattern of $\mu$. The largest correction is the one to $\mu_{33}$ for which $Y_{L 3}=Y_{R 3}=Y_{3}=1$. Taking $h \sim 0.1 \mathrm{MeV}$ (the value inferred from eq. (2.15) for $\left\langle\chi_{R}^{0}\right\rangle \simeq 10^{5} \mathrm{GeV}$ ) we obtain $\Delta \mu_{33} \sim 10 \mathrm{eV}$. The tree-level entries of the $\mu$ matrix are $\sim 0.1 \mathrm{MeV}$ in order to reproduce $\sim 0.1 \mathrm{eV}$ neutrino masses. Thus, the radiative corrections are much smaller than the tree-level contribution and the structure imposed by symmetries is preserved with high accuracy. 


\section{Phenomenology and naturalness}

\subsection{Heavy neutral lepton searches}

Neglecting the linear seesaw contribution, we obtain from eq. (2.9) the mass matrix in $\left(\nu_{L}, N_{L}, S^{c}\right)$ basis

$$
\mathcal{M}=\left(\begin{array}{ccc}
0 & m_{D} & 0 \\
m_{D} & 0 & M_{D} \\
0 & M_{D} & \mu
\end{array}\right)
$$

where both $m_{D}$ and $M_{D}$ are simultaneously diagonal under the screening assumption, and furthermore $m_{D i}=\xi M_{D i}$. The diagonalization of $\mathcal{M}$ can be performed in several steps.

(i). We start with rotation in the $\nu$-S plane

$$
\mathcal{U}_{S}=\left(\begin{array}{ccc}
c_{\xi} \mathbb{1} & 0 & s_{\xi} \mathbb{1} \\
0 & \mathbb{1} & 0 \\
-s_{\xi} \mathbb{1} & 0 & c_{\xi} \mathbb{1}
\end{array}\right)
$$

where

$$
s_{\xi}=\frac{\xi}{\sqrt{1+\xi^{2}}} \approx \xi
$$

and for brevity hereafter we denote $c \equiv \cos , s \equiv \sin$. After this rotation, in the new basis $\left(\nu^{\prime}, N_{L}, S^{\prime}\right)$, the 1-2 and 2-1 blocks vanish and the mass matrix becomes

$$
\mathcal{M}_{S}=\left(\begin{array}{ccc}
s_{\xi}^{2} \mu & 0 & -s_{\xi} c_{\xi} \mu \\
0 & 0 & M_{\xi} \\
-s_{\xi} c_{\xi} \mu & M_{\xi} & c_{\xi}^{2} \mu
\end{array}\right),
$$

with

$$
M_{\xi i} \equiv M_{D i} \sqrt{1+\xi^{2}} .
$$

(ii). Next, we perform rotations in the $N_{L}-S^{\prime}$ plane

$$
\mathcal{U}_{N}=\left(\begin{array}{ccc}
\mathbb{1} & 0 & 0 \\
0 & \mathbf{c}_{N} \mathbb{1} & \mathbf{s}_{N} \mathbb{1} \\
0 & -\mathbf{s}_{N} \mathbb{1} & \mathbf{c}_{N} \mathbb{1}
\end{array}\right),
$$

by the angles close to $45^{\circ}$ which approximately diagonalize the $N_{L^{-}} S^{\prime}$ block. Here $\mathbf{s}_{N} \equiv$ $\operatorname{diag}\left(s_{N}^{1}, s_{N}^{2}, s_{N}^{3}\right)$ and

$$
s_{N}^{i} \approx \frac{1}{\sqrt{2}}\left[1-\frac{\mu_{i i}}{4 M_{D i}}\right]
$$

where $\mu_{i i}(i=1,2,3)$ are the diagonal elements of the matrix $\mu$ and we take $c_{\xi} \simeq 1$. As a result, the mass matrix in the new basis $\left(\nu^{\prime}, N^{-}, N^{+}\right)$reads

$$
\mathcal{M}_{N} \approx\left(\begin{array}{ccc}
s_{\xi}^{2} \mu & \frac{1}{\sqrt{2}} s_{\xi} \mu-\frac{1}{\sqrt{2}} s_{\xi} \mu \\
\frac{1}{\sqrt{2}} s_{\xi} \mu & M^{-} & 0 \\
-\frac{1}{\sqrt{2}} s_{\xi} \mu & 0 & M^{+}
\end{array}\right) .
$$


Here,

$$
M_{i}^{-}=-M_{\xi_{i}}+\frac{1}{2} \mu_{i i}, \quad M_{i}^{+}=M_{\xi_{i}}+\frac{1}{2} \mu_{i i},
$$

are the masses of $N_{i}^{-}$and $N_{i}^{+}$. Thus, the fields $N_{i}^{-}$and $N_{i}^{+}$form a pair of quasi-degenerate heavy neutral leptons with $\sim \mu_{i i}$ mass splitting. For the phenomenology of heavy states, the 1-2 and 1-3 blocks in eq. (3.8) can be neglected.

(iii). Finally, we diagonalize the 1-1 block of the matrix (3.8), i.e. the light neutrino mass matrix, via

$$
\mathcal{U}_{\nu}=\left(\begin{array}{ccc}
U_{\nu} & 0 & 0 \\
0 & \mathbb{1} & 0 \\
0 & 0 & \mathbb{1}
\end{array}\right) .
$$

The transition to the flavor basis requires an additional rotation which diagonalizes the mass matrix of the charged leptons

$$
\mathcal{U}_{l}=\left(\begin{array}{lll}
U_{l} & 0 & 0 \\
0 & \mathbb{1} & 0 \\
0 & 0 & \mathbb{1}
\end{array}\right)
$$

Then, the total mixing matrix in the flavor basis is given by the product of rotations

$$
\mathcal{U}_{f}=\mathcal{U}_{l}^{\dagger} \mathcal{U}_{S} \mathcal{U}_{N} \mathcal{U}_{\nu}=\left(\begin{array}{ccc}
U_{\mathrm{PMNS}} & -\mathbf{s}_{N} s_{\xi} U_{l}^{\dagger} \mathbf{c}_{N} s_{\xi} U_{l}^{\dagger} \\
0 & \frac{1}{\sqrt{2}} \mathbb{1} & \frac{1}{\sqrt{2}} \mathbb{1} \\
-s_{\xi} U_{\nu} & -\mathbf{s}_{N} \mathbb{1} & \mathbf{c}_{N} \mathbb{1}
\end{array}\right)
$$

Neglecting $\mu$ contribution in eq. (3.7) and taking $c_{N}=s_{N}=1 / \sqrt{2}$ yields

$$
\nu_{\alpha}=U_{\mathrm{PMNS}} \nu-\frac{1}{\sqrt{2}} s_{\xi} U_{l}^{\dagger}\left(N^{-}-N^{+}\right),
$$

where $\nu_{\alpha}$ and $\nu$ are the flavor and light mass eigenstates, respectively. Since $U_{l}$ is nondiagonal, each active neutrino state has admixtures of all pairs of heavy leptons. These admixtures can be constrained by various terrestrial experiments as well as by cosmology [36]. In figure 3 we show the bounds (adopted from ref. [37]) on the admixtures of $N_{i}^{ \pm}(i=1,2,3)$ in $\left(\nu_{e}, \nu_{\mu}, \nu_{\tau}\right)$. Although these bounds have been derived for the mixing of a single heavy lepton, they are also applicable in our scenario where several heavy states are simultaneously present in the model.

According to eq. (3.13), the admixture of $N_{i}^{-}$and $N_{i}^{+}$in $\nu_{\alpha}$ equals

$$
\left|U_{\alpha i}^{N}\right|^{2} \equiv\left|U_{\alpha i}^{N^{-}}\right|^{2}=\left|U_{\alpha i}^{N^{+}}\right|^{2}=\frac{1}{2} s_{\xi}^{2}\left|U_{l \alpha i}\right|^{2}=\frac{1}{2}\left(\frac{m_{D i}}{M_{i}}\right)^{2}\left|U_{l \alpha i}\right|^{2}
$$

where in the last equality we expressed $s_{\xi}$ in terms of $N^{ \pm}$mass, and we define $M_{i}=$ $\left(M_{i}^{+}-M_{i}^{-}\right) / 2$. For relevant cases of our model, the production coherence of the mass eigenstates $N_{i}^{+}$and $N_{i}^{-}$is strongly broken, especially for the lightest leptons $(i=1)$ that appear in low energy processes. That is, $N_{i}^{+}$and $N_{i}^{-}$are produced (as components of N) 
and then decay incoherently without interference effects. Consequently, equal number of $l^{+}$ and $l^{-}$leptons will appear in the decays. For heavier leptons produced in very high energy processes (e.g. decays of $W_{R}$ ) the coherence can be maintained (see [14] and references therein). The experimental bounds, given in figure 3, are obtained for a production of a single heavy lepton. Since we deal here with two nearly degenerate states that are indistinguishable in experiments, the corresponding bounds on the individual mixing are two times stronger. In other words, we can treat the pair as a single particle and multiply the mixing by a factor of two so that the black lines in figure 3 correspond to

$$
2\left|U_{\alpha i}^{N}\right|^{2}=\frac{m_{D i}^{2}}{M_{i}^{2}}\left|U_{l \alpha i}\right|^{2}
$$

We use $m_{D i}^{2}=\left(m_{u}^{2}, m_{c}^{2}, m_{t}^{2}\right), U_{l \alpha i}=\left(V_{\mathrm{CKM}}\right)_{\alpha i}$ and do not impose here any relations between the heavy lepton masses $M_{i}$, in other words we are treating them as independent (the relaxation of the screening condition is discussed in section 4).

Notice that since $U_{l} \sim V_{\mathrm{CKM}} \sim \mathbb{1}$, the strongest bounds appear in the cases when the diagonal elements of $U_{l}$ are involved. The most stringent bound on the mass of $N_{1}^{ \pm}$comes from its admixture in $\nu_{e}$

$$
\left|U_{e 1}^{N}\right|^{2} \approx \frac{1}{2}\left(\frac{m_{D 1}}{M_{1}}\right)^{2} \approx \frac{m_{u}^{2}}{2 M_{1}^{2}}
$$

where $m_{u} \approx(1-2) \mathrm{MeV}$ is the mass of the up quark at the TeV scale. In the left panel of figure 3 we show with a black line (dashed in the excluded parameter space, solid elsewhere) the dependence of $2\left|U_{e 1}^{N}\right|^{2}$ on $M_{1}$ for $m_{u} \simeq 2 \mathrm{MeV}$. From this figure we infer

$$
M_{1} \geq 2 \mathrm{GeV},
$$

which is set by the CHARM $[38,39]$ exclusion region. Varying $m_{u}$ by a factor of 3 does not change the limit in eq. (3.17). However, for $m_{u}<0.5 \mathrm{MeV}$ the limit becomes weaker: $M_{1} \geq 0.4 \mathrm{GeV}$.

Despite the involved CKM suppression, the admixture of $N_{1}^{ \pm}$in $\nu_{\mu}$ yields practically identical (3.17) bound on $M_{1}$, which mainly stems from $\mathrm{NuTeV}$ [40]. From eq. (3.14) we have

$$
\left|U_{\mu 1}^{N}\right|^{2} \approx \frac{1}{2}\left(\frac{m_{D 1}}{M_{1}^{2}}\right)^{2}\left|U_{l \mu 1}\right|^{2} \approx \frac{m_{u}^{2}}{2 M_{1}^{2}} \sin ^{2} \theta_{\mathrm{c}},
$$

where $\theta_{\mathrm{c}}$ is the Cabibbo angle. In turn, this gives the bound

$$
\xi \leq 10^{-3} .
$$

In our framework, the neutral lepton masses are related by screening and the $q$ - $l$ similarity. Employing the limit (3.17) we obtain $M_{2}=M_{1} m_{c} / m_{u} \geq 600 \mathrm{GeV}$, where $m_{c} \approx 0.5 \mathrm{GeV}$ is the mass of the charm quark at the TeV scale [41]. From the screening relation for the mass of the third generation of heavy leptons, $M_{3}=M_{1} m_{t} / m_{u}$, we find the limit on the L-R symmetry breaking scale

$$
\left\langle\chi_{R}^{0}\right\rangle \approx \sqrt{2} M_{3} \geq 2 \times 10^{5} \mathrm{GeV} .
$$




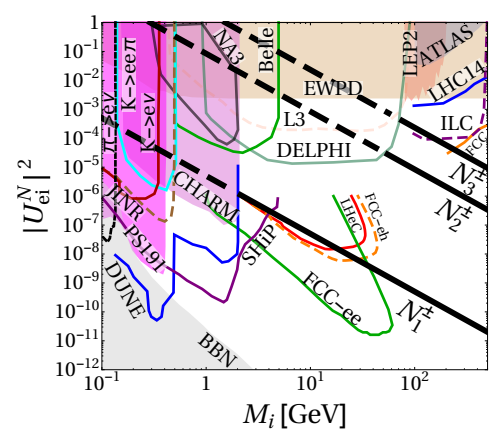

(a)

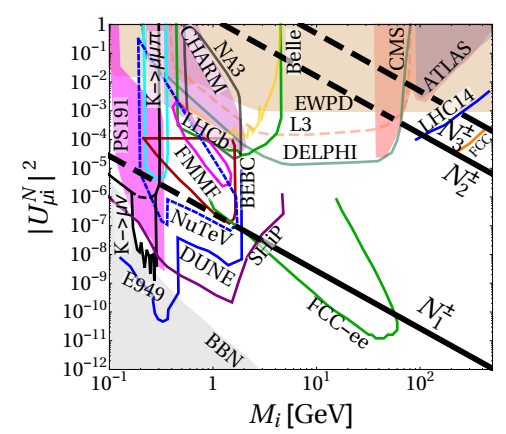

(b)

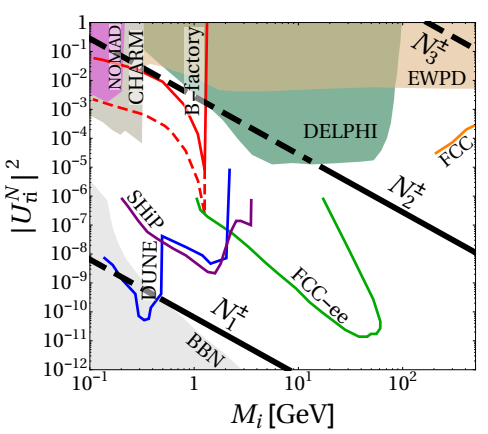

(c)

Figure 3. Experimental bounds on and future sensitivities to the heavy lepton mixing in $\nu_{e}$ (panel (a)), $\nu_{\mu}$ (panel (b)) and $\nu_{\tau}$ (panel (c)). The black lines show the predicted mixing of heavy leptons $N_{i}^{ \pm}(i=1,2,3)$ in a given light neutrino flavor state as a function of $M_{i}$. We take $\left(Y_{1}, Y_{2}, Y_{3}\right) \simeq\left(2 \times 10^{-5}, 0.007,1\right)$. Solid (dashed) parts of the black lines are expectations in the allowed (excluded) region.

The condition in eq. (3.20) makes discovery of the $\mathrm{RH}$ gauge bosons and scalars at present colliders unfeasible. The leptons $N_{2}^{ \pm}$and $N_{3}^{ \pm}$are beyond the reach of current and future experiments as well (see the lines in all panels of figure 3). However, as can be seen from the left and middle panel of figure 3 , the lightest pair of neutral leptons is accessible to future colliders [42-44], beam-dump experiments [45] and neutrino oscillation facilities [46]. In particular, SHiP may improve the lower bound (3.17) on $M_{1}$ to approximately $5 \mathrm{GeV}$, whereas FCC-ee will be able to probe even larger masses (up to $60 \mathrm{GeV}$ ) and very tiny mixing angles. Hadron collider FCC-hh with the total center of mass energy around $100 \mathrm{TeV}$ will be able to search for $N_{2}^{ \pm}$(see line denoted $F C C$ in all panels of figure 3) and also $N_{3}^{ \pm}$ in the absence of screening [47], as well as the $\mathrm{RH}$ gauge bosons and new scalar bosons from the Higgs doublets [48].

Without imposing the screening condition, the strongest direct bound on the mass of $N_{2}^{ \pm}$follows from its admixture in $\nu_{\mu}$

$$
\left|U_{\mu 2}^{N}\right|^{2} \approx \frac{1}{2}\left(\frac{m_{D 2}}{M_{2}}\right)^{2} \approx \frac{m_{c}^{2}}{2 M_{2}^{2}} .
$$

The regions excluded by DELPHI [49] and CMS [50] experiments give $M_{2}>70 \mathrm{GeV}$ (see $N_{2}^{ \pm}$line in the middle panel of figure 3 ). Decreasing $m_{D 2}$ by a factor of 3 relaxes the bound down to $M_{2}>40 \mathrm{GeV}$.

The admixture of $N_{2}^{ \pm}$in $\nu_{e}$ is characterized by

$$
\left|U_{e 2}^{N}\right|^{2} \approx \frac{1}{2}\left(\frac{m_{D 2}}{M_{2}}\right)^{2} \sin ^{2} \theta_{c} \approx \frac{m_{c}^{2}}{2 M_{2}^{2}} \sin ^{2} \theta_{c},
$$

and according to figure 3 (left panel) this leads to $M_{2}>50 \mathrm{GeV}$.

The strongest direct bound on the mass of $N_{3}^{ \pm}, M_{3}>100 \mathrm{GeV}$, is established by ATLAS. Note that the direct bounds on $N_{2}^{ \pm}$and $N_{3}^{ \pm}$are significantly weaker than those obtained from the bound on $N_{1}^{ \pm}$and the screening relation (see eqs. (3.17) and (3.20)). 
Thus, in the absence of screening, the hierarchy of the heavy leptons can be much weaker. We will elaborate on such a scenario in section 4.

Let us note that the neutral leptons, $\left(N^{+}+N^{-}\right) / \sqrt{2}$, are produced dominantly via the mixing with active neutrinos in the processes involving the left-handed gauge bosons [51]. The production of $N^{-}$and $N^{+}$via the exchange of the off-shell right-handed bosons in $e^{+} e^{-}, p \bar{p}$ and $p p$ collisions is subdominant as the corresponding cross-sections are suppressed by a factor $\xi^{4}=\left(\left\langle\phi_{1}^{0}\right\rangle /\left\langle\chi_{R}^{0}\right\rangle\right)^{4}$.

\section{$3.20 \nu 2 \beta$ decay}

The dominant contribution to the neutrinoless double beta $(0 \nu 2 \beta)$ decay arises from the left-handed current since the right-handed current contribution scales as $\xi^{4} \lesssim 10^{-12}$ [52-54] due to the bound (3.19). Then, the effective Majorana mass of the electron neutrino can be presented as

$$
m_{e e}=m_{e e}^{l}+m_{e e}^{h},
$$

where the contributions from the light and heavy mass eigenstates read

$$
\begin{aligned}
& m_{e e}^{l}=\sum_{i=1,2,3}\left(U_{\text {PMNS } e i}\right)^{2} m_{i} \approx s_{\xi}^{2} \mu_{e e} \\
& m_{e e}^{h}=r p^{2} \sum_{i=1,2,3}\left(\left(U_{e i}^{N^{-}}\right)^{2} \frac{M_{i}}{p^{2}-M_{i}^{2}}+\left(U_{e i}^{N^{+}}\right)^{2} \frac{M_{i}^{\prime}}{p^{2}-M_{i}^{\prime 2}}\right) \equiv \sum_{i=1,2,3} m_{i}^{h} .
\end{aligned}
$$

Here, $r \equiv M_{\beta \beta 0 \nu}^{l} / M_{\beta \beta 0 \nu}^{h}$ is the ratio of nuclear matrix elements for the exchange of heavy and light neutrinos, $-p^{2} \sim(125 \mathrm{MeV})^{2}$ is the neutrino momentum squared and $\mu_{e e}$ denotes the $(1,1)$ element of the $\mu$ matrix.

The contribution from the $i$-th pair of pseudo-Dirac neutrinos $\left(N_{i}^{-}\right.$and $\left.N_{i}^{+}\right)$can be estimated as

$$
m_{i}^{h}=\xi^{2} \mu_{e e} \frac{p^{2}}{M_{i}^{2}} \sim m_{e e}^{l} \frac{p^{2}}{M_{i}^{2}},
$$

where $\xi^{2}$ arises from the admixture of $N_{i}^{ \pm}$in $\nu_{e}$ and $\mu_{e e}$ stems from the sum $M_{i}^{+}+M_{i}^{-}$ (see eq. (3.9)). Due to strong mass hierarchy, the contributions from the heavier pairs are negligible and only the lightest one $\left(N_{1}^{-}\right.$and $\left.N_{1}^{+}\right)$should be considered. Hence, $m_{e e}^{h} \approx m_{1}^{h}$.

To compute $m_{1}^{h}$ one should retain the 1-2 and 1-3 blocks in the matrix (3.8) and perform further rotations to diagonalize it up to $\mathcal{O}\left(\mu^{2}\right)$. The terms proportional to the elements of $\mu$ matrix can not be neglected and, in particular, deviations of the $N_{L}-S$ mixing from $45^{\circ}$ should be taken into account in eq. (3.12). To diagonalize the matrix in eq. (3.8) we perform two additional rotations:

$$
\mathcal{U}_{14}=\left(\begin{array}{ccc}
\mathbb{1} & U_{14} & 0 \\
-U_{14} & \mathbb{1} & 0 \\
0 & 0 & \mathbb{1}
\end{array}\right), \quad \mathcal{U}_{17}=\left(\begin{array}{ccc}
\mathbb{1} & 0 & U_{17} \\
0 & \mathbb{1} & 0 \\
-U_{17} & 0 & \mathbb{1}
\end{array}\right)
$$

with

$$
U_{14} \approx U_{17}=\left(\begin{array}{ccc}
-\frac{s_{\xi} c_{\xi}}{\sqrt{2}} \frac{\mu_{e e}}{M_{1}} & 0 & 0 \\
0 & 0 & 0 \\
0 & 0 & 0
\end{array}\right)
$$


Now the total mixing matrix equals

$$
\mathcal{U}_{f}=\mathcal{U}_{l}^{\dagger} \mathcal{U}_{S} \mathcal{U}_{N} \mathcal{U}_{\nu} \mathcal{U}_{14} \mathcal{U}_{17}
$$

According to eq. (3.28), the flavor neutrino states can be expressed in terms of mass eigenstates

$$
\nu_{f}=c_{\xi} U_{\mathrm{PMNS}} \nu+\left(c_{\xi} U_{\mathrm{PMNS}} U_{14}-s_{\xi} s_{N} U_{l}^{\dagger}\right) N_{1}^{-}+\left(c_{\xi} U_{\mathrm{PMNS}} U_{17}+s_{\xi} c_{N} U_{l}^{\dagger}\right) N_{1}^{+},
$$

where we neglected the small correction to the first term. Then, the admixtures of $N_{1}^{-}$ and $N_{1}^{+}$in $\nu_{e}$ are given explicitly by

$$
\mp \frac{s_{\xi}}{\sqrt{2}}\left[1 \pm \frac{c_{\xi}^{2} \mu_{e e}}{M_{1}}\left(\left(U_{\mathrm{PMNS}}\right)_{e 1}-\frac{1}{4}\left(U_{l}^{\dagger}\right)_{e 1}\right)\right],
$$

where the upper (lower) sign corresponds to $U_{e 1}^{N^{-}}\left(U_{e 1}^{N^{+}}\right)$. After inserting these expressions into eq. (3.24), we find

$$
m_{1}^{h}=-2\left(U_{\mathrm{PMNS}}\right)_{e 1}\left(U_{l}^{\dagger}\right)_{e 1} \frac{\left|p^{2}\right|}{M_{1}^{2}} s_{\xi}^{2} c_{\xi}^{2} \mu_{e e}=-2\left(U_{\mathrm{PMNS}}\right)_{e 1}\left(U_{l}^{\dagger}\right)_{e 1} \frac{\left|p^{2}\right|}{M_{1}^{2}} m_{e e}^{l} .
$$

Thus, the mass $m_{1}^{h}$ is proportional to the contribution from light neutrinos, $m_{e e}^{l}$, with the additional suppression factor $\left|p^{2}\right| / M_{1}^{2}$, in agreement with the estimate in eq. (3.25). The ratio of the two contributions equals

$$
\frac{m_{1}^{h}}{m_{e e}^{l}}=1.67 \frac{\left|p^{2}\right|}{M_{1}^{2}} .
$$

For $M_{1}=0.4 \mathrm{GeV}$ and $M_{1}=2 \mathrm{GeV}$ this suppression is 0.1 and 0.004 , respectively. Hence, the heavy lepton contribution is practically not observable in the $0 \nu 2 \beta$ decay experiments.

\subsection{Leptogenesis}

The inverse seesaw mechanism with its pairs of quasi-degenerate heavy leptons appears, at a first sight, very suitable for realization of low scale leptogenesis mechanisms. Those include the resonant leptogenesis [55, 56], where the lepton asymmetry is produced from the decays of quasi-degenerate heavy leptons, and the ARS mechanism based on oscillations between heavy neutral leptons [57]. A vast majority of the previous studies on leptogenesis in the inverse seesaw framework have been performed in the SM gauge structure. In the case of L-R model with the $q-l$ similarity we have large Yukawa couplings and additional processes which enhance the washout effect.

In its minimal setup, the inverse seesaw model is not compatible with successful thermal leptogenesis due to strong washout $[58,59]$. The maximal achievable value of the baryon asymmetry is 8 orders of magnitude smaller than the observed one [60].

In our L-R symmetric setup, similar conclusions apply. Furthermore, what already prevents any possibility for viable leptogenesis in our model is the size of the Yukawa 
couplings chosen according to the $q-l$ similarity. As a result, the out-of-equilibrium condition is not satisfied for all pairs of heavy leptons. In particular, for the second pair with $M_{2} \sim \mathrm{TeV}$ there is no deviation from thermal equilibrium for couplings $Y_{2} \gtrsim \mathcal{O}\left(10^{-7}\right)$. For $Y_{2} \lesssim \mathcal{O}\left(10^{-7}\right)$ we obtain $\left(N^{ \pm}-N^{\mathrm{eq}}\right) / N_{\text {eq }} \gtrsim 10^{-1}$ at $M / T \gtrsim \mathcal{O}(1)$. This should be compared with $Y_{2} \sim 5 \cdot 10^{-3}$ in our model.

The observed baryon asymmetry can be produced in the non-minimal realization of the inverse seesaw $[60,61]$ which is motivated by explanation of the scale of Majorana masses $\mu$. The extension includes an extra fermion singlet $X$ with large Majorana mass $M_{X} \gg M_{i}$ and the Higgs singlet $\sigma$ which develops a VEV. The authors [60] employ the additional global symmetry under which $S$ is charged. This is, however, not possible in our scenario since $S, \sigma$ and $X$ are not charged under any global symmetry.

The leptogenesis mechanisms discussed so far either fail in producing the required amount of asymmetry or are not compatible with our model setup. For alternative and potentially successful low-scale leptogenesis scenarios (see below) it is necessary to avoid strong washout. At $T \sim M_{N}$, the lepton number violating process $L_{L} \Phi \rightarrow L_{L}^{c} \Phi^{\dagger}$ mediated by the Majorana fermions $N^{-}$and $N^{+}$becomes operative. The strength of the washout is quantified by the parameter $K_{i}$ which, for a given fermion generation $i$, reads

$$
\begin{array}{lr}
\frac{\Gamma_{i}}{H\left(T=M_{i}\right)}\left(\frac{\mu_{i}}{\Gamma_{i}}\right)^{2}, & \text { for } \mu \ll \Gamma_{i}, \\
\frac{\Gamma_{i}}{H\left(T=M_{i}\right)}, & \text { for } \mu \geq \Gamma_{i} .
\end{array}
$$

Here, $\Gamma_{i}=Y_{i}^{2} M_{i} /(8 \pi)$ is the decay rate of $N_{i}^{ \pm}$. The additional factor $\left(\mu_{i} / \Gamma_{i}\right)^{2}$ in eq. (3.33a) originates from the interference between diagrams containing quasi-degenerate particles $\left(N^{-}\right.$and $\left.N^{+}\right)[62,63]$.

Let us make general estimations by dropping the assumptions of the $q$ - $l$ similarity and screening. From the inverse seesaw formula we have $\mu=2 m_{\nu} M^{2} /\left(Y^{2}\left\langle\phi_{1}^{0}\right\rangle^{2}\right)$, and consequently

$$
\frac{\mu}{\Gamma}=\frac{16 \pi m_{\nu}}{\left\langle\phi_{1}^{0}\right\rangle^{2}} \frac{M}{Y^{4}}=8.3 \cdot 10^{-9} \frac{1}{Y^{4}}\left(\frac{m_{\nu}}{0.1 \mathrm{eV}}\right)\left(\frac{M}{10^{5} \mathrm{GeV}}\right),
$$

where for brevity we omitted flavor generation indices. For $m_{\nu}=0.01 \mathrm{eV}, M=200 \mathrm{GeV}$ and $Y=1.1 \cdot 10^{-3}$, we obtain $\mu / \Gamma=1$. For smaller $Y$, we should use expression (3.33b) which gives $K \gg 1$, implying a very strong washout unless $Y \lesssim \mathcal{O}\left(10^{-7}\right)$. For $Y>1.1 \cdot 10^{-3}$, the expression (3.33a) should be used. It can be rewritten as

$$
K=32 \pi \frac{m_{\nu}^{2} M}{Y^{6} a\left\langle\phi_{1}^{0}\right\rangle^{4}} \simeq 1.95 \cdot 10^{-5} \frac{1}{Y^{6}}\left(\frac{M}{10^{5} \mathrm{GeV}}\right)\left(\frac{m_{\nu}}{0.1 \mathrm{eV}}\right)^{2},
$$

where $a=1.66 \sqrt{g_{*}} / M_{\mathrm{Pl}}$. The condition $K<1$ gives even stronger bound on the coupling, namely $Y>0.06$ for $M>200 \mathrm{GeV}$. Together with the bound on the admixture of the heavy leptons in the flavor states $\xi=Y v_{L} / M<10^{-2}$ (which is not applicable above TeV scale) this leads to a narrow range of the allowed parameters: $M=\left(10^{3}-10^{5}\right) \mathrm{GeV}$ and $Y>0.1$, which is satisfied only for the third generation. 
For the inverse seesaw, the ARS mechanism leads to successful leptogenesis if $Y \sim$ $10^{-7}-10^{-6}$ [64]. For masses of $N^{-}$and $N^{+}$at $\mathcal{O}(\mathrm{TeV})$ the washout (3.33b) is suppressed. Note, however, that such parameter space is not in accord with $q-l$ similarity.

One can rely on the electroweak baryogenesis [65] which requires a strong first order electroweak phase transition. The rich scalar sector in our model may help to realize such a scenario. Still, the lepton number washout should be suppressed, since sphalerons partially carry the baryon asymmetry to the lepton section.

\subsection{Corrections to the Higgs mass. Naturalness}

In this section we will address a specific problem related to existence of heavy $\mathrm{RH}$ neutrinos (recall that for the third neutrino $M_{3} \gg v_{E W}$ ) and their radiative corrections to the Higgs mass. It was noticed long time ago that the contribution to the Higgs mass from the loops formed by active neutrinos and heavy RH neutrinos in the type I seesaw mechanism equals [66] (see [67-69] for recent studies)

$$
\delta m_{H}^{2} \approx \frac{Y_{i}^{2} M_{i}^{2}}{4 \pi^{2}}=\frac{m_{\nu 3} M_{3}^{3}}{2 \pi^{2}\left\langle\phi_{1}^{0}\right\rangle^{2}}
$$

Then, the condition that the correction $\delta m_{H}^{2}$ (3.36) does not exceed the Higgs mass itself ("naturalness"), $\delta m_{H}^{2} \lesssim 10^{4} \mathrm{GeV}^{2}$, leads to the upper bound on the mass [66]

$$
M_{i} \lesssim \text { few } \times 10^{7} \mathrm{GeV},
$$

where $m_{\nu 3} \sim 0.1 \mathrm{eV}$ was used. This is smaller than the standard lower bound from thermal leptogenesis [70] (however, see [71]).

With $M_{i} \leq 10^{5} \mathrm{GeV}$ (that we obtained in section 3.1), the bound (3.37) is satisfied. However, in the inverse seesaw, the correction to $\delta m_{H}^{2}$ becomes larger than in the type I seesaw model, being enhanced by the factor $M_{i} / \mu[72]$

$$
\delta m_{H}^{2} \sim \frac{m_{\nu 3} M_{i}^{4}}{2 \pi^{2} \mu_{\max }\left\langle\phi_{1}^{0}\right\rangle^{2}} .
$$

Here, $\mu_{\max }$ represents the largest entry of the $\mu$ matrix. The difference from expression (3.36) originates from different dependence of the neutrino mass on the mass of heavy leptons. Eq. (3.38) and the inequality $\delta m_{H}^{2}<10^{4} \mathrm{GeV}^{2}$ give the bound $M_{i}<10^{4} \mathrm{GeV}$ for $\mu_{\max } \sim \mathcal{O}(100) \mathrm{keV}$. This bound is about 1 order of magnitude lower than $M_{3}$. At the same time, in our scenario (in contrast to, e.g., ref. [66]), there are other particles, gauge bosons and scalars, at the scale $10^{5} \mathrm{GeV}$. Being bosons, these particles give corrections to the Higgs mass of the opposite sign and they can cancel contributions from the RH neutrinos. Let us consider such a possibility.

For the gauge boson contribution we consider the 1-loop corrections with heavy righthanded charged $\left(W_{R}^{ \pm}\right)$and neutral $\left(Z_{R}\right)$ vector bosons. To estimate $\delta m_{H}^{2}$ arising from the loops of $W_{R}^{ \pm}, Z_{R}$ and $N_{3}^{ \pm}$we use the 1-loop effective potential [73, 74]. Expanding the 1-loop potential and identifying the term containing two SM Higgs fields we obtain 
the condition required for a 1-loop cancellation between the fermion and gauge boson contributions to $\delta m_{H}^{2}$

$$
\frac{Y_{3}^{2}}{g^{2}} \simeq\left(\frac{11}{8}\right)^{1 / 2} \approx 1.17
$$

In eq. (3.39), $g$ is the $\mathrm{SU}(2)$ gauge coupling. In our framework, Yukawa couplings are fixed according to the $q-l$ similarity condition and the gauge coupling for $\mathrm{SU}(2)_{R}$ equals the $\mathrm{SU}(2)_{L}$ one. The values of $g$ and $Y_{3}$ at the top quark mass scale are 0.65 and 0.93 , respectively, so that $Y_{3}^{2} / g^{2}=2.05$ which clearly fails to satisfy eq. (3.39). Even though the renormalization group effects may help a bit (we find $7 \%$ decrease of $Y_{3} / g$ at $\left\langle\chi_{R}^{0}\right\rangle \sim 10^{5} \mathrm{GeV}$ ) the cancellation can not be achieved without additional scalar contribution. Another possibility is that $Y_{3}$ differs from the top Yukawa coupling.

In the scalar sector a number of diagrams can contribute to the SM Higgs mass. If only one scalar with mass $m^{2} \sim(c / 2)\left\langle\chi_{R}^{0}\right\rangle^{2}$ gives the dominant contribution to $\delta m_{H}^{2}$, the condition for complete cancellation of bosonic and fermionic 1-loop contributions to the SM Higgs mass reads

$$
11 g^{4}-8 Y_{3}^{4}+2 c^{2} \simeq 0 .
$$

Note that for this cancellation one needs specific values of couplings in the scalar sector. There is no principle which would fix the couplings to such values implying that if the cancellation occurs it is accidental. If the full 1-loop cancellation is achieved, the dominant Higgs mass correction stems from 2-loop diagrams which can be estimated as [75]

$$
\delta m_{H}^{2} \sim \frac{g^{2} Y_{3}^{2}}{\left(16 \pi^{2}\right)^{2}} M_{3}^{2} \approx \frac{g^{2}}{2\left(16 \pi^{2}\right)^{2}}\left\langle\chi_{R}^{0}\right\rangle^{2}=3.4 \cdot 10^{5} \mathrm{GeV}^{2} .
$$

The value given in eq. (3.41) is only one order of magnitude larger than $m_{H}^{2}$, and therefore a rather acceptable level of fine-tuning is required. Note again that a small deviation from the $q-l$ similarity can also solve the problem.

Let us underline that in our scenario new physics scale is just 3 orders of magnitude larger than the EW scale and hence we deal here with mild hierarchy in the scalar sector. We do not discuss the origin of this hierarchy, but we find that the hierarchy is supported by the price of moderate fine tuning. The hierarchy can be further weakened if we depart from the q-1 similarity.

Of course, in the complete analysis one should take into account all corrections to the Higgs mass including corrections from the top quark. Note that in the dimensional regularization the leading corrections are proportional to the mass of particle propagating in the loop. Therefore, the correction from top and other EW scale particles are much smaller that those from heavy right-handed neutrinos.

\section{Variation on the theme}

\subsection{Altering heavy fermion portal couplings}

In the scenario described in section 3.1 the bound on the scale of L-R symmetry breaking has been obtained using the following three points: $(i)$ the lower bound on the mass of 
the lightest heavy leptons $N_{1}^{ \pm}$(see eq. (3.17)), (ii) the screening (2.23), and (iii) the $q$ $l$ similarity, eq. (2.27). The latter implies the equalities $M_{3}=M_{1} m_{t} / m_{u}$ and $Y_{3}=1$. Consequently,

$$
\left\langle\chi_{R}^{0}\right\rangle \simeq \sqrt{2} M_{3}=\sqrt{2} M_{1} \frac{m_{t}}{m_{u}} .
$$

The scale of $\left\langle\chi_{R}^{0}\right\rangle$ can be reduced if the assumptions in eq. (2.23) and/or eq. (2.27) are relaxed. Let us discuss the two scenarios in which one of these assumptions is abandoned.

(i) Departing from the $\boldsymbol{q}-\boldsymbol{l}$ similarity. We keep the screening which can be expressed as

$$
Y_{i}=R_{0} Y_{R i}
$$

where in general $R_{0} \neq 1$. This implies

$$
\xi_{i} \equiv \frac{m_{D i}}{M_{D i}}=\frac{\left\langle\phi_{1}^{0}\right\rangle}{\left\langle\chi_{R}^{0}\right\rangle} \frac{Y_{i}}{Y_{R i}}=\frac{\left\langle\phi_{1}^{0}\right\rangle}{\left\langle\chi_{R}^{0}\right\rangle} R_{0} \equiv \xi
$$

i.e. all $\xi_{i}$ are equal and eq. (2.24) for $m_{\nu}$ is unchanged. Let us recall that $\xi$ determines admixtures of heavy leptons in the flavor states. In the absence of the $q-l$ similarity, the Dirac masses $m_{D i}$ are free parameters.

The strongest bound on $\xi$ is obtained from mixing of $N_{1}^{ \pm}$in $\nu_{e}$ since $\xi^{2}=\left|U_{e 1}^{N}\right|^{2}$. From figure 3 one finds $\xi \leq 0.006$ and $M_{1}>2 \mathrm{GeV}$. This corresponds to $m_{D 1} \leq 12 \mathrm{MeV}$.

As the Dirac masses $m_{D i}$ are not fixed, even the extreme scenario with all $m_{D i}$ (and therefore $M_{i}$ ) being equal is possible. For $m_{D i}=12 \mathrm{MeV}$ all the heavy leptons are accessible to experiments. From the expression for the light neutrino masses (2.24), we find that for $\xi=0.006$ and $m_{\nu 3}=0.05 \mathrm{eV}$ the entries of the Majorana mass $\mu$ should be $\mathcal{O}(1) \mathrm{keV}$.

The scale of L-R symmetry breaking can be substantially lowered in comparison to the value given in eq. (3.20). Namely, the bound can be as low as the bound from the direct $\mathrm{RH}$ gauge boson searches, which is roughly $3 \mathrm{TeV}[50,76]$.

(ii) Departing from the exact screening. Now we keep the $q-l$ similarity only. In this case, $\xi_{i} \equiv m_{D i} / M_{D i}$ are different. Taking weaker hierarchy of $M_{i}$ than that of $m_{D i}$, for instance $M_{i}=\left(2,200,5 \cdot 10^{3}\right) \mathrm{GeV}$, we have $\xi_{i}=\left(1.15 \cdot 10^{-3}, 6.45 \cdot 10^{-3}, 3.45 \cdot 10^{-2}\right)$. The corresponding effective mixing parameters given by $\left|\xi_{i}\right|^{2}$ yield

$$
2\left|U_{e 1}^{N}\right|^{2} \simeq 1.32 \cdot 10^{-6}, \quad 2\left|U_{\mu 2}^{N}\right|^{2} \simeq 4.16 \cdot 10^{-5}, \quad 2\left|U_{\tau 3}^{N}\right|^{2} \simeq 1.18 \cdot 10^{-3} .
$$

Now, $\left\langle\chi_{R}^{0}\right\rangle=\sqrt{2} M_{3} \simeq 7 \mathrm{TeV}$ for $Y_{R 3} \simeq 1$, so that both additional neutral leptons and right-handed gauge bosons are accessible to LHC.

Due to departure from screening, the formula for light neutrino masses changes

$$
m_{\nu}=\left(\frac{\left\langle\phi_{1}^{0}\right\rangle}{\left\langle\chi_{R}^{0}\right\rangle}\right)^{2} R \mu R, \quad R \equiv \operatorname{diag}\left(Y_{1} / Y_{R 1}, Y_{2} / Y_{R 2}, Y_{3} / Y_{R 3}\right),
$$

where $R$ can be absorbed in redefinition of the matrix $\mu$ :

$$
\mu_{i j} \rightarrow \mu_{i j}^{\prime}=\mu_{i j} R_{i} R_{j} .
$$

Numerically, for chosen $M_{i}$ we obtain $R \simeq(0.033,0.185,1)$. 
The appearance of $R$ may, however, complicate the explanation of mixing pattern from symmetry arguments since now both $Y$ and $Y_{R}$ are non-trivially involved in the expression for the light neutrino mass matrix. Consequently, certain correlation between $R$ and $\mu$ matrices should exist. Also, $R$ would affect phenomenology of the heavy leptons (production, decay, etc.).

\subsection{Left and right fermion singlets}

So far we considered the scenario with single Majorana fermion $S$ per generation, that is the common fermion $S$ for the left and right sectors. This is consistent with L-R symmetry. Under $P$ transformations we had $S_{L} \leftrightarrow\left(S^{c}\right)_{R}$. Minimal and logically straighforward extension of this scenario is a P-symmetric model with two independent singlets $S_{L}$ and $S_{R}$ for the left and the right sectors, respectively. This study allows to check wheather the L-R symmetry can be realized in the singlet sector.

Now the Yukawa interactions and mass terms read

$$
\begin{aligned}
\mathcal{L} \supset & -\bar{L}_{R} Y \Phi^{\dagger} L_{L}-\bar{L}_{R} \tilde{Y} \tilde{\Phi}^{\dagger} L_{L}-\bar{S}_{L}^{c} Y_{L} \tilde{\chi}_{L}^{\dagger} L_{L}-\bar{S}_{R}^{c} Y_{R} \tilde{\chi}_{R}^{\dagger} L_{R} \\
& -\frac{1}{2}\left[\bar{S}_{L}^{c} \mu_{L L} S_{L}+\bar{S}_{R}^{c} \mu_{R R} S_{R}+\bar{S}_{L} \mu_{L R} S_{R}\right]+\text { h.c. }
\end{aligned}
$$

Due to P-invariance, the relations (2.8) involving $Y$ and $Y_{L(R)}$ still hold as before. Now, the Yukawa interactions in (4.7) are invariant with respect to global U(1) symmetry of the lepton number with charge prescription $L_{g}\left(L_{L}\right)=L_{g}\left(L_{R}\right)=1, L_{g}\left(S_{L}\right)=L_{g}\left(S_{R}\right)=$ -1 , and zero charges for scalar fields. This symmetry forbids the Yukawa interactions $\bar{S}_{R(L)} \tilde{\chi}_{L(R)}^{\dagger} L_{L(R)}$. The symmetry is broken by the Majorana mass terms (second line in eq. (4.7)). The smallness of masses $\mu$ can be related to this breaking.

Invariance with respect to $P$ transformation $S_{L} \leftrightarrow S_{R}$ would imply the following equalities: $\mu_{L L}=\mu_{R R}, \mu_{L R}=\mu_{L R}^{\dagger}$. However, as in the visible sector, masses can break parity, so that in general $\mu_{L L} \neq \mu_{R R}$. The breaking can be spontaneous if $\mu$ terms are generated by couplings of $S$ with singlet scalars $\sigma_{L}, \sigma_{R} \sigma_{L R}: y_{L} \bar{S}_{L}^{c} S_{L} \sigma_{L}, y_{R} \bar{S}_{R}^{c} S_{R} \sigma_{R}$. Then, even if we impose P-symmetry which gives $y_{R}=y_{L}$, the scales of $\mu_{L L}$ and $\mu_{R R}$ can be different due to $\left\langle\sigma_{L}\right\rangle \neq\left\langle\sigma_{R}\right\rangle$, i.e. spontaneous violation of parity in the singlet sector. In this case $\mu_{L L} \propto \mu_{R R}$. With complicated singlet sector one can obtain also different structures of matrices $\mu_{L L}$ and $\mu_{R R}$. Finally, the L-R symmetry may be explicitly broken in the S-sector. In what follows we will not specify origins of $\mu$ matrices, but consider $a$ priori general structure of $\mu_{L L}$ and $\mu_{L R}$ assuming only that $\mu \ll v_{E W}$. As before, $\mu_{R R}$ is fixed via inverse seesaw by masses and mixing of light active neutrinos. Notice that in this extension the contribution to the $\chi_{L}^{\dagger} \tilde{\Phi} \chi_{R}$ coupling is generated by the loop diagram of figure 1 with $\mu$ substituted by $\mu_{L R}$.

After the Higgs fields acquire VEVs, the mass matrix in the $\left(\nu_{L}, N_{L}, S_{L}, S_{R}^{c}\right)$ basis reads

$$
\mathcal{M}=\left(\begin{array}{cccc}
0 & m_{D} & m_{D}^{\prime} & 0 \\
m_{D} & 0 & 0 & M_{D} \\
m_{D}^{\prime} & 0 & \mu_{L L} & \mu_{L R} \\
0 & M_{D} & \mu_{L R}^{T} & \mu_{R R}
\end{array}\right)
$$


where all entries are $3 \times 3$ matrices and the expressions for $m_{D}, m_{D}^{\prime}$ and $M_{D}$ are given in eq. (2.10). The procedure of diagonalization of this matrix is similar to the one outlined in section 3.1. First, we make a rotation in the $\nu_{L}-S_{R}^{c}$ plane by $s_{\xi}$. In the new basis $\left(\nu^{\prime}, N_{L}, S_{L}, S^{\prime}\right)$ we perform nearly maximal rotation in the $\left(N_{L}-S^{\prime}\right)$ plane. Then, in the rotated basis $\left(\nu^{\prime}, N^{-}, S_{L}, N^{+}\right), N^{-}$and $S_{L}$ are permuted and the pseudo-Dirac states $\left(N^{-}\right.$and $\left.N^{+}\right)$decouple. In the basis of light states $\left(\nu^{\prime}, S_{L}\right)$ the mass matrix reads

$$
\left(\begin{array}{cc}
\mu_{R R} s_{\xi}^{2} & c_{\xi} m_{D}^{\prime}-s_{\xi} \mu_{L R} \\
c_{\xi} m_{D}^{\prime}-s_{\xi} \mu_{L R} & \mu_{L L}
\end{array}\right) .
$$

Interestingly, the decoupling of $N^{-}$and $N^{+}$does not produce $\mathcal{O}\left(\mu_{i j} / M_{D}\right)$ corrections to this matrix.

Phenomenology of this extended version is largely identical to the one of the main scenario. In particular, properties of heavy pseudo-Dirac states formed now by $\nu_{R}$ and $S_{R}$ are similar to ones presented in section 3.1. The light neutrino mass matrix is given by the $(1,1)$ element of $(4.9), \mu_{R R} s_{\xi}^{2}$, and the observed values of the neutrino masses are achieved for $\mu_{R R} \sim 10 \mathrm{keV}$ and $s_{\xi}^{2} \lesssim 10^{-6}$.

The only substantial difference from the main scenario is the presence of three relatively light states $S_{L}$. Therefore, in what follows we will focus on new physics associated to $S_{L}$.

According to (4.7), the states $S_{L}$ have Yukawa interactions with the left leptonic doublet $L_{L}$ and heavy scalar doublet $\chi_{L}(4.7)$. The mass of $\chi_{L}$ is at the $\mathrm{SU}(2)_{R}$ symmetry breaking scale and for estimations we will use $M_{\chi}=3 \cdot 10^{5} \mathrm{GeV}$. The Yukawa couplings $Y_{L}$ are large, being $Y_{3} \sim 1$ for the third generation. Furthermore, $S_{L}$ mix with light (mostly active) neutrinos according to eq. (4.9). The mixing angles with light neutrinos are given by

$$
\sin \theta_{S i} \approx \frac{1}{\mu_{L L i}}\left(c_{\xi} m_{D i}^{\prime}-s_{\xi} \mu_{L R i}\right)
$$

where for simplicity we have taken the matrices $m_{D}^{\prime}, \mu_{L L}$ and $\mu_{L R}$ to be diagonal. In the case of full cancellation in the above expression, $S_{L}$ states and light neutrinos do not mix. The mixing gives additional contribution to the light neutrino masses

$$
\delta m_{\nu} \approx \sin ^{2} \theta_{S i} m_{L L i}
$$

Therefore, the condition that there is no significant contribution to the light neutrino masses from mixing with $S_{L}$ gives the upper bound

$$
\sin ^{2} \theta_{S i} \ll \frac{m_{\nu}}{m_{L L i}},
$$

where $m_{\nu} \sim(0.01-0.02) \mathrm{eV}$.

The states $S_{L}$ are light sterile neutrinos and their properties (masses, mixing and interactions) are subject to strong cosmological bounds. At the same time, $S_{L}$ can be a dark matter candidate or even a very light particle in meV-eV mass range with no observable contributions to the energy density of the Universe. 
The states $S_{L}$ decay into three light neutrinos, $S_{i L} \rightarrow \nu \nu \bar{\nu}$, via mixing with light neutrinos and $Z^{0}$ exchange. In vacuum, the lifetime equals

$$
\tau_{i}=3.8 \cdot 10^{20} \sec \left(\frac{10^{-6}}{\sin ^{2} \theta_{S i}}\right)\left(\frac{10 \mathrm{keV}}{\mu_{L L i}}\right)^{5} .
$$

Notice that, according to (4.12), for $\mu_{L L i}=10 \mathrm{keV}$ and $\sin ^{2} \theta_{S i}=10^{-6}$ the contribution to the light masses equals $\sim 0.01 \mathrm{eV}$.

The heavier $S_{i L}$ can also decay into lighter $S_{j L}$ with the $\chi^{0}$ exchange: $S_{j L} \rightarrow S_{i L} \nu \bar{\nu}$. Typical time for this mode is

$$
\frac{1}{\Gamma_{i}}=2.4 \cdot 10^{27} \sec \left(\frac{10 \mathrm{keV}}{\mu_{L L i}}\right)^{5}\left(\frac{M_{\chi}}{3 \cdot 10^{5} \mathrm{GeV}}\right)^{4} \frac{1}{Y_{j}^{2} Y_{i}^{2}},
$$

which is much bigger than the decay time (4.13) via process where mixing in employed.

Thus, the lifetime of $S_{L}$ with masses (10-100) keV is much larger than the age of the Universe. Therefore, these $S_{L}$ can be candidates for the Dark matter particles if their appropriate number density is generated [77-83].

$S_{i L}$ can be produced via mixing with active neutrinos and oscillations. In this case the conditions on parameters of $S_{i L}$ to be a dark matter are similar to those in $\nu$ MSM [81]. For masses in the ballpark of $10 \mathrm{keV}$, the mixing should to be $\sin ^{2} \theta_{S} \lesssim 2.5 \cdot 10^{-11}$. The strongest limits on $\theta_{S}$ arise from X-ray searches [84, 85], Supernova 1987A [86, 87] and structure formation [88].

According to eq. (4.10), for $m_{D}^{\prime}=0$

$$
\sin \theta_{S} \approx-s_{\xi} \frac{\mu_{L R}}{\mu} .
$$

Then, for $s_{\xi} \simeq 10^{-3}$ eq. (4.15) yields $\mu_{L R} \lesssim 10^{-2} \mu$.

In addition, in our scenario $S_{i L}$ can be produced in the process $\nu_{i} \bar{\nu}_{j} \rightarrow S_{k L} \bar{S}_{l L}$ with $\chi_{L}$-exchange. Here, mixing is kept, so that $S_{k L}$ and $S_{l L}$ are the eigenstates of $\mu_{L L}$ matrix (with mixing due to $\mu_{L R}$ being neglected). Suppression of rate of this process, $\Gamma_{S}$, with respect to the rate of usual active neutrino reactions, $\Gamma_{\nu}$, is

$$
\frac{\Gamma_{S}}{\Gamma_{\nu}}=\frac{4}{g^{4}} Y_{i L}^{2} Y_{j L}^{2}\left|U_{i k}\right|^{2}\left|U_{j l}\right|^{2}\left(\frac{m_{W}}{M_{\chi}}\right)^{4} \approx 1.14 \cdot 10^{-13} Y_{i L}^{2} Y_{j L}^{2}\left|U_{i k}\right|^{2}\left|U_{j l}\right|^{2}\left(\frac{3 \cdot 10^{5} \mathrm{GeV}}{M_{\chi}}\right)^{4}
$$

where $U_{i k}$ is the mixing matrix elements of $S_{k L}$ and $g$ is the weak coupling constant.

Depending on values of masses and mixing of $S_{k L}$ one can consider different possibilities. Let us consider two extreme cases.

1. If the L-R symmetry holds in the singlet sector, then mixing between $S_{L}$ states is the same as the one for $S_{R}$, which is of the TBM type. Since the mixing is large, the ratio of rates (4.16) will be determined by the largest coupling $Y_{3 L}=1$ and the mixing matrix elements $U_{3 k}=U_{\tau k}^{\mathrm{TBM}}$. If $S_{2 L}$ is in the $10 \mathrm{keV}$ range and other $S_{L}$ states are lighter, then $\left|U_{32}\right|^{2}=1 / 6$ and from (4.16) we obtain the ratio of rates $\Gamma_{S} / \Gamma_{\nu}=3 \cdot 10^{-15}$. Using this ratio we find that $S_{L}$ exits the equilibrium with thermal bath at temperatures $T \sim 100 \mathrm{GeV}$. 
Their density will be diluted due to decrease of number of degrees of freedom at lower temperatures. This dilution is, however, not enough and further suppression by factor of 30 is needed to match correct energy density of dark matter. This can be achieved if the reheating temperature, $T_{\text {reh }}$, after inflation is below $100 \mathrm{GeV}$. Similar situation is for $S_{3 L}$ and $S_{1 L}$ which do not contribute substantially to the present energy density in the Universe due to smaller masses.

With such low $T_{\text {reh}}$, however, it will be difficult to realize baryogenesis through leptogenesis, unless $M_{\chi}$ increases or the coupling $Y_{L}$ decreases leading to higher $S_{L}$ decoupling temperature.

2. If mixing in the $S_{L}$ sector is absent, the DM component, taken to be $S_{1 L}$, will have the smallest coupling $Y_{1 L}=10^{-5}$. (This scenario can be reconciled with spontaneous L-R symmetry introducing additional flavons $\sigma$ ). In this case, the ratio of rates (4.16) equals $\approx 10^{-13}\left|Y_{1 L}\right|^{2}=10^{-23}$. Correspondingly, the decoupling (freeze-out) temperature will be $\sim 10^{5} \mathrm{GeV}$ and the required number density of $S_{1 L}$ can be obtained at $T_{\text {reh }} \sim 10^{4} \mathrm{GeV}$. This is much higher than in the previous case, and opens a possibility for low scale baryogenesis through leptogenesis.

Notice that in view of problems with generation of the number density via mixing in $\nu \mathrm{MSM}[81]$, the $\chi_{L^{-}}$-exchange can be the main mechanism of the DM production, while the mixing is suppressed sufficiently to satisfy the bounds from $X$-ray observations.

\section{Summary}

The low scale left-right symmetric models accessible to the existing and planned colliders are at odds with generation of naturally small neutrino masses. The (1-100) TeV scale of the L-R symmetry breaking (and consequently, the scale of RH neutrino masses) requires very small Dirac masses of neutrinos which strongly break the natural condition of quark-lepton similarity $Y \sim Y_{q}$. This similarity can be retained using the inverse seesaw mechanism which requires introduction of three fermionic singlets as well as the left and the right handed Higgs doublets. These doublets break the L-R symmetry and provide the portal for interactions of singlets with the SM particles.

This setup allows to obtain the neutrino mixing of special form e.g. tribimaximal or bimaximal. Under the screening condition, $Y_{R}=Y$, the light neutrino mass matrix is proportional to the Majorana mass matrix $\mu$ of singlet fermions $S$. In turn, special form of $\mu$ can be governed by symmetry in the S-sector. This symmetry is generally broken by the other interactions in the model but we have shown that the corrections due to such breaking are small and do not destroy the structure of $\mu$.

The contribution from the linear seesaw should be suppressed. If dominant, it would require unnaturally small Dirac neutrino mass terms with the structure that breaks the $q-l$ similarity. Such suppression can be achieved by small VEV of the left-handed doublet.

The generic consequence of the inverse seesaw is the existence of three heavy pseudoDirac neutral leptons with a mass splitting of the order of $\mu$. Under the conditions of $q-l$ similarity and screening, these heavy leptons mix with active neutrinos with strength 
$\xi \simeq\left\langle\phi_{1}^{0}\right\rangle /\left\langle\chi_{R}^{0}\right\rangle$ and have strongly hierarchical mass spectrum. Consequently, only the lightest states, $N_{1}^{ \pm}$, are accessible to current and near-future experiments. From the present experimental searches, we obtain the bound on mass of $N_{1}^{ \pm}$to be $M_{1} \geq(0.4-2) \mathrm{GeV}$. This, in turn, leads to the lower bound on the scale of L-R symmetry breaking $\left\langle\chi_{R}^{0}\right\rangle>$ $200 \mathrm{TeV}$. Future experiments such as SHiP, DUNE and FCC-ee can strengthen these bounds significantly. The FCC-hh collider will be able to test the existence of other new particles: $N_{2}^{ \pm}, N_{3}^{ \pm}$, the RH gauge bosons and new scalars.

Contributions of the heavy leptons to the effective Majorana mass $m_{e e}$ are suppressed, being proportional to the contributions of light states.

The leptogenesis scenarios do not yield the required amount of the baryon asymmetry in our model, primarily due to strong washout. However, with a certain extensions of the model (addition of new heavy fermion(s) $X$ and another singlet $S$ per generation) or departure from $q-l$ similarity (ARS), the required lepton asymmetry can be produced. The electroweak baryogenesis is a viable option.

The corrections to the Higgs mass $\left(\delta m_{H}^{2}\right)$, induced via loops of $\mathrm{RH}$ neutrinos are, for $\left\langle\chi_{R}^{0}\right\rangle=200 \mathrm{TeV}$, about 4 orders of magnitude larger than the experimentally observed value $\left(m_{H}^{2} \sim 10^{4} \mathrm{GeV}^{2}\right)$. The contributions from gauge boson loops as well as additional scalars can lead to substantial reduction of this correction. We derived the conditions for the complete cancellation of the Higgs mass one-loop corrections. Admittedly, such cancellations would be accidental.

Finally, we considered a scenario with two singlets per generation: $S_{L}$ in the left sector and $S_{R}$ in the right sector. In such a case the L-R symmetry is explicit and furthermore the global lepton number can be introduced. This modification leads to the appearance of $\mathrm{keV}$-scale leptons and the lightest of them can play the role of dark matter.

\section{Acknowledgments}

We are greatly indebted to Evgeny Akhmedov, Giorgio Arcadi, Arindam Das, Bhupal Dev, Peizhi Du, Oliver Fischer, Jisuke Kubo, Goran Senjanović and Xun-Jie Xu for several very useful discussions.

Open Access. This article is distributed under the terms of the Creative Commons Attribution License (CC-BY 4.0), which permits any use, distribution and reproduction in any medium, provided the original author(s) and source are credited.

\section{References}

[1] J.C. Pati and A. Salam, Lepton number as the fourth color, Phys. Rev. D 10 (1974) 275 [Erratum ibid. D 11 (1975) 703] [INSPIRE].

[2] R.N. Mohapatra and J.C. Pati, Left-right gauge symmetry and an isoconjugate model of CP-violation, Phys. Rev. D 11 (1975) 566 [inSPIRE].

[3] R.N. Mohapatra and J.C. Pati, A natural left-right symmetry, Phys. Rev. D 11 (1975) 2558 [INSPIRE]. 
[4] G. Senjanović and R.N. Mohapatra, Exact left-right symmetry and spontaneous violation of parity, Phys. Rev. D 12 (1975) 1502 [INSPIRE].

[5] R.N. Mohapatra and D.P. Sidhu, Gauge theories of weak interactions with left-right symmetry and the structure of neutral currents, Phys. Rev. D 16 (1977) 2843 [INSPIRE].

[6] G. Senjanović, Is left-right symmetry the key?, Mod. Phys. Lett. A 32 (2017) 1730004 [arXiv: 1610.04209] [INSPIRE].

[7] P.S. Bhupal Dev, S. Goswami and M. Mitra, TeV scale left-right symmetry and large mixing effects in neutrinoless double beta decay, Phys. Rev. D 91 (2015) 113004 [arXiv:1405.1399] [INSPIRE].

[8] M. Lindner, F.S. Queiroz, W. Rodejohann and C.E. Yaguna, Left-right symmetry and lepton number violation at the large hadron electron collider, JHEP 06 (2016) 140 [arXiv: 1604.08596] [INSPIRE].

[9] P.S.B. Dev, R.N. Mohapatra and Y. Zhang, Probing the Higgs sector of the minimal left-right symmetric model at future hadron colliders, JHEP 05 (2016) 174 [arXiv: 1602.05947] [INSPIRE].

[10] P.S.B. Dev and R.N. Mohapatra, TeV scale inverse seesaw in $\mathrm{SO}(10)$ and leptonic non-unitarity effects, Phys. Rev. D 81 (2010) 013001 [arXiv:0910.3924] [InSPIRE].

[11] V. Tello, M. Nemevšek, F. Nesti, G. Senjanović and F. Vissani, Left-right symmetry: from LHC to neutrinoless double beta decay, Phys. Rev. Lett. 106 (2011) 151801 [arXiv: 1011.3522] [INSPIRE].

[12] Y. Zhang, H. An, X. Ji and R.N. Mohapatra, General CP-violation in minimal left-right symmetric model and constraints on the right-handed scale, Nucl. Phys. B 802 (2008) 247 [arXiv: 0712.4218] [INSPIRE].

[13] A. Das, N. Nagata and N. Okada, Testing the 2 TeV resonance with trileptons, JHEP 03 (2016) 049 [arXiv: 1601.05079] [inSPIRE].

[14] A. Das, P.S.B. Dev and R.N. Mohapatra, Same sign versus opposite sign dileptons as a probe of low scale seesaw mechanisms, Phys. Rev. D 97 (2018) 015018 [arXiv:1709.06553] [INSPIRE].

[15] P. Fileviez Perez, C. Murgui and S. Ohmer, Simple left-right theory: lepton number violation at the LHC, Phys. Rev. D 94 (2016) 051701 [arXiv:1607.00246] [InSPIRE].

[16] P. Minkowski, $\mu \rightarrow e \gamma$ at a rate of one out of $10^{9}$ muon decays?, Phys. Lett. B 67 (1977) 421 [INSPIRE].

[17] M. Gell-Mann, P. Ramond and R. Slansky, Complex spinors and unified theories, Conf. Proc. C 790927 (1979) 315 [arXiv:1306.4669] [InSPIRE].

[18] T. Yanagida, Horizontal gauge symmetry and masses of neutrinos, Conf. Proc. C $\mathbf{7 9 0 2 1 3 1}$ (1979) 95 [INSPIRE].

[19] R.N. Mohapatra and G. Senjanović, Neutrino mass and spontaneous parity nonconservation, Phys. Rev. Lett. 44 (1980) 912 [INSPIRE].

[20] R.N. Mohapatra and J.W.F. Valle, Neutrino mass and baryon number nonconservation in superstring models, Phys. Rev. D 34 (1986) 1642 [INSPIRE].

[21] R.N. Mohapatra, Mechanism for understanding small neutrino mass in superstring theories, Phys. Rev. Lett. 56 (1986) 561 [INSPIRE]. 
[22] P.-H. Gu and U. Sarkar, Leptogenesis with linear, inverse or double seesaw, Phys. Lett. B 694 (2011) 226 [arXiv: 1007.2323] [INSPIRE].

[23] E.K. Akhmedov, M. Lindner, E. Schnapka and J.W.F. Valle, Left-right symmetry breaking in NJLS approach, Phys. Lett. B 368 (1996) 270 [hep-ph/9507275] [INSPIRE].

[24] D. Wyler and L. Wolfenstein, Massless neutrinos in left-right symmetric models, Nucl. Phys. B 218 (1983) 205 [INSPIRE].

[25] M. Lindner, M.A. Schmidt and A. Yu. Smirnov, Screening of Dirac flavor structure in the seesaw and neutrino mixing, JHEP 07 (2005) 048 [hep-ph/0505067] [INSPIRE].

[26] A.Y. Smirnov and X.-J. Xu, Neutrino mixing in $\mathrm{SO}(10)$ GUTs with a non-Abelian flavor symmetry in the hidden sector, Phys. Rev. D 97 (2018) 095030 [arXiv:1803.07933] [INSPIRE].

[27] G. Senjanović, Spontaneous breakdown of parity in a class of gauge theories, Nucl. Phys. B 153 (1979) 334 [INSPIRE].

[28] P.S. Bhupal Dev, R.N. Mohapatra, W. Rodejohann and X.-J. Xu, Vacuum structure of the left-right symmetric model, arXiv:1811.06869 [INSPIRE].

[29] N.G. Deshpande, J.F. Gunion, B. Kayser and F.I. Olness, Left-right symmetric electroweak models with triplet Higgs, Phys. Rev. D 44 (1991) 837 [INSPIRE].

[30] G. 't Hooft, Naturalness, chiral symmetry, and spontaneous chiral symmetry breaking, NATO Sci. Ser. B 59 (1980) 135 [InSPIRE].

[31] P.F. Harrison, D.H. Perkins and W.G. Scott, Tri-bimaximal mixing and the neutrino oscillation data, Phys. Lett. B 530 (2002) 167 [hep-ph/0202074] [INSPIRE].

[32] F. Vissani, A study of the scenario with nearly degenerate Majorana neutrinos, hep-ph/9708483 [INSPIRE].

[33] V.D. Barger, S. Pakvasa, T.J. Weiler and K. Whisnant, Bimaximal mixing of three neutrinos, Phys. Lett. B 437 (1998) 107 [hep-ph/9806387] [INSPIRE].

[34] P.O. Ludl and A. Yu. Smirnov, Lepton mixing from the hidden sector, Phys. Rev. D 92 (2015) 073010 [arXiv: 1507.03494] [INSPIRE].

[35] A. Yu. Smirnov, Seesaw enhancement of lepton mixing, Phys. Rev. D 48 (1993) 3264 [hep-ph/9304205] [INSPIRE].

[36] A.C. Vincent, E.F. Martinez, P. Hernández, M. Lattanzi and O. Mena, Revisiting cosmological bounds on sterile neutrinos, JCAP 04 (2015) 006 [arXiv: 1408.1956] [INSPIRE].

[37] F.F. Deppisch, P.S. Bhupal Dev and A. Pilaftsis, Neutrinos and collider physics, New J. Phys. 17 (2015) 075019 [arXiv: 1502.06541] [INSPIRE].

[38] CHARM collaboration, A search for decays of heavy neutrinos in the mass range $0.5 \mathrm{GeV}$ to 2.8 GeV, Phys. Lett. B 166 (1986) 473 [INSPIRE].

[39] J. Orloff, A.N. Rozanov and C. Santoni, Limits on the mixing of tau neutrino to heavy neutrinos, Phys. Lett. B 550 (2002) 8 [hep-ph/0208075] [INSPIRE].

[40] NuTEV and E815 collaborations, Search for neutral heavy leptons in a high-energy neutrino beam, Phys. Rev. Lett. 83 (1999) 4943 [hep-ex/9908011] [INSPIRE].

[41] Z.-Z. Xing, H. Zhang and S. Zhou, Updated values of running quark and lepton masses, Phys. Rev. D 77 (2008) 113016 [arXiv:0712.1419] [InSPIRE]. 
[42] S. Antusch, E. Cazzato and O. Fischer, Displaced vertex searches for sterile neutrinos at future lepton colliders, JHEP 12 (2016) 007 [arXiv: 1604.02420] [INSPIRE].

[43] D. d'Enterria, Physics at the FCC-ee, in Proceedings, $17^{\text {th }}$ Lomonosov Conference on Elementary Particle Physics, Moscow, Russia, 20-26 August 2015, World Scientific, Singapore (2017), pg. 182 [arXiv: 1602.05043] [INSPIRE].

[44] FCC-ee Study Team collaboration, Search for heavy right handed neutrinos at the FCC-ee, Nucl. Part. Phys. Proc. 273-275 (2016) 1883 [arXiv:1411.5230] [INSPIRE].

[45] O. Lantwin, Search for new physics with the SHiP experiment at CERN, PoS (EPS-HEP2017) 304 (2017) [arXiv: 1710. 03277] [INSPIRE].

[46] DUNE collaboration, Long-Baseline Neutrino Facility (LBNF) and Deep Underground Neutrino Experiment (DUNE), arXiv:1601.05471 [INSPIRE].

[47] S. Antusch, E. Cazzato and O. Fischer, Sterile neutrino searches at future $e^{-} e^{+}, p p$ and $e^{-} p$ colliders, Int. J. Mod. Phys. A 32 (2017) 1750078 [arXiv:1612.02728] [inSPIRE].

[48] T. Golling et al., Physics at a $100 \mathrm{TeV}$ pp collider: beyond the Standard Model phenomena, CERN Yellow Report (2017) 441 [arXiv:1606.00947] [INSPIRE].

[49] DELPHI collaboration, Search for neutral heavy leptons produced in $Z$ decays, Z. Phys. C 74 (1997) 57 [Erratum ibid. C 75 (1997) 580] [INSPIRE].

[50] CMS collaboration, Search for heavy neutrinos and $W$ bosons with right-handed couplings in proton-proton collisions at $\sqrt{s}=8 \mathrm{TeV}$, Eur. Phys. J. C 74 (2014) 3149 [arXiv:1407.3683] [INSPIRE].

[51] S. Banerjee, P.S.B. Dev, A. Ibarra, T. Mandal and M. Mitra, Prospects of heavy neutrino searches at future lepton colliders, Phys. Rev. D 92 (2015) 075002 [arXiv:1503.05491] [INSPIRE].

[52] P.S. Bhupal Dev, S. Goswami, M. Mitra and W. Rodejohann, Constraining neutrino mass from neutrinoless double beta decay, Phys. Rev. D 88 (2013) 091301 [arXiv:1305.0056] [INSPIRE].

[53] H. Päs and W. Rodejohann, Neutrinoless double beta decay, New J. Phys. 17 (2015) 115010 [arXiv: 1507.00170] [INSPIRE].

[54] F.F. Deppisch, C. Hati, S. Patra, P. Pritimita and U. Sarkar, Neutrinoless double beta decay in left-right symmetric models with a universal seesaw mechanism, Phys. Rev. D 97 (2018) 035005 [arXiv: 1701.02107] [INSPIRE].

[55] A. Pilaftsis and T.E.J. Underwood, Resonant leptogenesis, Nucl. Phys. B 692 (2004) 303 [hep-ph/0309342] [INSPIRE].

[56] A. De Simone and A. Riotto, On resonant leptogenesis, JCAP 08 (2007) 013 [arXiv:0705.2183] [INSPIRE].

[57] E.K. Akhmedov, V.A. Rubakov and A. Yu. Smirnov, Baryogenesis via neutrino oscillations, Phys. Rev. Lett. 81 (1998) 1359 [hep-ph/9803255] [INSPIRE].

[58] P.S. Bhupal Dev, P. Millington, A. Pilaftsis and D. Teresi, Flavour covariant transport equations: an application to resonant leptogenesis, Nucl. Phys. B 886 (2014) 569 [arXiv: 1404.1003] [INSPIRE].

[59] B. Dev, M. Garny, J. Klaric, P. Millington and D. Teresi, Resonant enhancement in leptogenesis, Int. J. Mod. Phys. A 33 (2018) 1842003 [arXiv:1711.02863] [InSPIRE]. 
[60] K. Agashe, P. Du, M. Ekhterachian, C.S. Fong, S. Hong and L. Vecchi, Hybrid seesaw leptogenesis and TeV singlets, Phys. Lett. B 785 (2018) 489 [arXiv: 1804.06847] [InSPIRE].

[61] M. Aoki, N. Haba and R. Takahashi, A model realizing inverse seesaw and resonant leptogenesis, PTEP 2015 (2015) 113B03 [arXiv:1506.06946] [INSPIRE].

[62] S. Blanchet, T. Hambye and F.-X. Josse-Michaux, Reconciling leptogenesis with observable $\mu \rightarrow$ er rates, JHEP 04 (2010) 023 [arXiv:0912.3153] [INSPIRE].

[63] S. Blanchet, P.S.B. Dev and R.N. Mohapatra, Leptogenesis with TeV scale inverse seesaw in SO(10), Phys. Rev. D 82 (2010) 115025 [arXiv: 1010.1471] [InSPIRE].

[64] A. Abada, G. Arcadi, V. Domcke and M. Lucente, Neutrino masses, leptogenesis and dark matter from small lepton number violation?, JCAP 12 (2017) 024 [arXiv: 1709.00415] [INSPIRE].

[65] D.E. Morrissey and M.J. Ramsey-Musolf, Electroweak baryogenesis, New J. Phys. 14 (2012) 125003 [arXiv: 1206.2942] [INSPIRE].

[66] F. Vissani, Do experiments suggest a hierarchy problem?, Phys. Rev. D 57 (1998) 7027 [hep-ph/9709409] [INSPIRE].

[67] F. Bazzocchi and M. Fabbrichesi, Little hierarchy problem for new physics just beyond the LHC, Phys. Rev. D 87 (2013) 036001 [arXiv:1212.5065] [INSPIRE].

[68] J.D. Clarke, R. Foot and R.R. Volkas, Electroweak naturalness in the three-flavor type-I seesaw model and implications for leptogenesis, Phys. Rev. D 91 (2015) 073009 [arXiv: 1502.01352] [INSPIRE].

[69] G. Bambhaniya, P. Bhupal Dev, S. Goswami, S. Khan and W. Rodejohann, Naturalness, vacuum stability and leptogenesis in the minimal seesaw model, Phys. Rev. D 95 (2017) 095016 [arXiv: 1611.03827] [INSPIRE].

[70] S. Davidson and A. Ibarra, A lower bound on the right-handed neutrino mass from leptogenesis, Phys. Lett. B 535 (2002) 25 [hep-ph/0202239] [INSPIRE].

[71] K. Moffat, S. Pascoli, S.T. Petcov, H. Schulz and J. Turner, Three-flavored nonresonant leptogenesis at intermediate scales, Phys. Rev. D 98 (2018) 015036 [arXiv:1804.05066] [INSPIRE].

[72] N. Haba, H. Ishida and Y. Yamaguchi, Naturalness and lepton number/flavor violation in inverse seesaw models, JHEP 11 (2016) 003 [arXiv: 1608.07447] [INSPIRE].

[73] S.R. Coleman and E.J. Weinberg, Radiative corrections as the origin of spontaneous symmetry breaking, Phys. Rev. D 7 (1973) 1888 [INSPIRE].

[74] J.A. Casas, V. Di Clemente, A. Ibarra and M. Quirós, Massive neutrinos and the Higgs mass window, Phys. Rev. D 62 (2000) 053005 [hep-ph/9904295] [INSPIRE].

[75] M. Fabbrichesi and A. Urbano, Naturalness redux: the case of the neutrino seesaw mechanism, Phys. Rev. D 92 (2015) 015028 [arXiv:1504.05403] [INSPIRE].

[76] S. Patra, F.S. Queiroz and W. Rodejohann, Stringent dilepton bounds on left-right models using LHC data, Phys. Lett. B 752 (2016) 186 [arXiv: 1506.03456] [INSPIRE].

[77] S. Dodelson and L.M. Widrow, Sterile-neutrinos as dark matter, Phys. Rev. Lett. 72 (1994) 17 [hep-ph/9303287] [INSPIRE].

[78] X.-D. Shi and G.M. Fuller, A new dark matter candidate: nonthermal sterile neutrinos, Phys. Rev. Lett. 82 (1999) 2832 [astro-ph/9810076] [INSPIRE]. 
[79] A. Merle, V. Niro and D. Schmidt, New production mechanism for keV sterile neutrino dark matter by decays of frozen-in scalars, JCAP 03 (2014) 028 [arXiv:1306.3996] [INSPIRE].

[80] V. Brdar, J. Kopp, J. Liu and X.-P. Wang, X-ray lines from dark matter annihilation at the keV scale, Phys. Rev. Lett. 120 (2018) 061301 [arXiv:1710.02146] [INSPIRE].

[81] T. Asaka and M. Shaposhnikov, The $\nu M S M$, dark matter and baryon asymmetry of the universe, Phys. Lett. B 620 (2005) 17 [hep-ph/0505013] [INSPIRE].

[82] T. Asaka, S. Blanchet and M. Shaposhnikov, The $\nu M S M$, dark matter and neutrino masses, Phys. Lett. B 631 (2005) 151 [hep-ph/0503065] [INSPIRE].

[83] S. Baumholzer, V. Brdar and P. Schwaller, The new $\nu M S M$ ( $\nu \nu M S M)$ : radiative neutrino masses, keV-scale dark matter and viable leptogenesis with sub-TeV new physics, JHEP 08 (2018) 067 [arXiv: 1806.06864] [INSPIRE].

[84] K. Perez, K.C.Y. Ng, J.F. Beacom, C. Hersh, S. Horiuchi and R. Krivonos, Almost closing the $\nu$ MSM sterile neutrino dark matter window with NuSTAR, Phys. Rev. D 95 (2017) 123002 [arXiv: 1609.00667] [INSPIRE].

[85] K. Abazajian, G.M. Fuller and W.H. Tucker, Direct detection of warm dark matter in the X-ray, Astrophys. J. 562 (2001) 593 [astro-ph/0106002] [INSPIRE].

[86] G.G. Raffelt and S. Zhou, Supernova bound on keV-mass sterile neutrinos reexamined, Phys. Rev. D 83 (2011) 093014 [arXiv: 1102.5124] [InSPIRE].

[87] C.A. Argüelles, V. Brdar and J. Kopp, Production of keV sterile neutrinos in supernovae: new constraints and gamma ray observables, arXiv:1605.00654 [INSPIRE].

[88] A. Schneider, Astrophysical constraints on resonantly produced sterile neutrino dark matter, JCAP 04 (2016) 059 [arXiv: 1601.07553] [INSPIRE]. 